\title{
A homography-based dynamic control approach applied to station keeping of autonomous underwater vehicles without linear velocity measurements
}

\author{
Lam-Hung Nguyen, Minh-Duc Hua, Guillaume Allibert and Tarek Hamel
}

\begin{abstract}
A homography-based dynamic control approach applied to station keeping of Autonomous Underwater Vehicles (AUVs) without relying on linear velocity measurements is proposed. The homography estimated from images of a planar target scene captured by a downward-looking camera is directly used as feedback information. The full dynamics of the AUV are exploited in a hierarchical control design with inner-outer loop architecture. Enhanced by integral compensation actions and disturbance torque estimation, the proposed controller is robust with respect to model uncertainties and unknown currents. The performance of the proposed control approach is illustrated via both comparative simulation results conducted on a realistic AUV model and experimental validations on an in-house AUV.
\end{abstract}

Index Terms-Autonomous Underwater Vehicle, visual servoing, homography-based control, station keeping, nonlinear control

\section{INTRODUCTION}

Safe and efficient navigation of Autonomous Underwater Vehicles (AUVs) in cluttered environments remains a challenging task. Scientific issues are particularly related to the fact that global acoustic positioning systems become unusable or insufficiently precise in such situations, leading to an obvious interest in developing advanced sensor-based control strategies for AUV applications in close proximity to a complex sea bottom or submarine structures. While acoustic systems have been widely used for sensing underwater environments, cameras offer an appealing alternative due to the rich information captured by images and their high update rate. By using vision sensors as a sensor modality for relative (scaled) position and orientation, the control problems can be cast into PositionBased Visual Servoing (PBVS) or Image-Based Visual Servoing (IBVS) [5]. Classical visual servo control techniques have been initially developed for robotic manipulators and mobile ground vehicles [15], [21] and then for aerial drones [7], [9], [10]. In underwater robotics, vision sensors have been used to perform station keeping or positioning [6], [8], [19], [31], docking [4], [20], [22], [32], and pipeline following [1], [2], [25], [30], etc.

Both stereo and monocular cameras have been exploited for stabilization and positioning of AUVs. When the vehicle's

The authors are with I3S laboratory (Laboratoire d'Informatique, Signaux et Systèmes de Sophia-Antipolis), CNRS, Université Côte d'Azur, UMR7271, Sophia Antipolis, France. T. Hamel is also with Institut Universitaire de France. Emails: Ihnguyen(hua,allibert,thamel)@i3s.unice.fr. pose (i.e. position and orientation) can be estimated, existing PBVS controllers can be directly applied [28]. In contrast, the case of monocular vision without the assumption of planarity of the visual target and the prior knowledge of its geometry is more involved since full pose reconstruction from visual data is not possible. However, monocular vision can be sufficient to achieve stabilization of an AUV in front of a planar target [4], [19], [31]. Recently, an advanced kinematic IBVS control scheme was proposed in [3] by exploiting the socalled homography that is an invertible mapping relating two camera views of the same planar scene by encoding in a single matrix the camera pose, the distance between the camera and the scene, and the normal direction to the scene [3], [11]. A remarkable feature of that approach is the non-requirement of homography decomposition often computationally expensive (see e.g. [24]), as opposed to other homography-based visual servo (HBVS) controllers [4], [23]. More recently, this kinematic HBVS control approach has been extended in our prior work [17] in order to account for the full dynamics of fully-actuated AUVs and to obtain an enlarged provable domain of stability. The present paper continues to extend our work [17] to the case where linear velocity measurements are unavailable. We restrict, however, our study to the case of a downward-looking camera. One of the main motivations behind this effort is related to the development of a low-cost but efficient solution for station keeping of AUVs without the need of a costly Doppler Velocity Log (DVL) velocity sensor. More precisely, the proposed solution makes use of a minimal and inexpensive sensor suite consisting of an Inertial Measurement Unit (IMU) and an embedded video camera whose total cost can be less than $150 €$. A similar problem concerning underactuated aerial drones addressed in [7] is in line with our effort in dealing with system's dynamics and in depleting the need of a linear velocity sensor. However, the approach proposed in [7] relies on the assumption that the visual velocity is available for control design, for instance, via the use of a high-gain observer, but a complete stability analysis including such high-gain observer is missing.

The paper is structured into seven sections including the introduction and concluding sections. Notation and problem formulation of HBVS control of fully-actuated AUVs are provided in Section III. Section IIII presents a recall on system modeling together with a simplified model for control design. In Section [V] a novel inertial-aided homography-based 
dynamic control approach applied to station keeping of fullyactuated AUVs without relying on linear velocity measurements is proposed. Comparative simulation results conducted on a realistic AUV model are reported in Section V, showing the performance and robustness of the proposed approach. Section VI first describes some practical development aspects of the experimental platform then reports extensive experimental validation results in a real environment. Three video links are also provided showing these experimental results.

A primary version of this work has been presented at a conference [27]. Several extensions of [27] are proposed in this paper. For instance, the restrictive assumption of alignment between the AUV's center of buoyancy and center of mass and the camera optical center used in [27] is no longer necessary, allowing one to accommodate any arbitrary camera location on the AUV. The inner-loop controller has been modified by using a bounded nested saturation integrator of the angular velocity error instead of a classical integrator that is prone to the well-known phenomenon of integrator wind-up. Finally, the comparative simulation study and extensive experimental validations on an in-house AUV of this paper have been newly developed.

\section{NotATION AND PROBLEM FORMULATION}

\section{A. Notation}

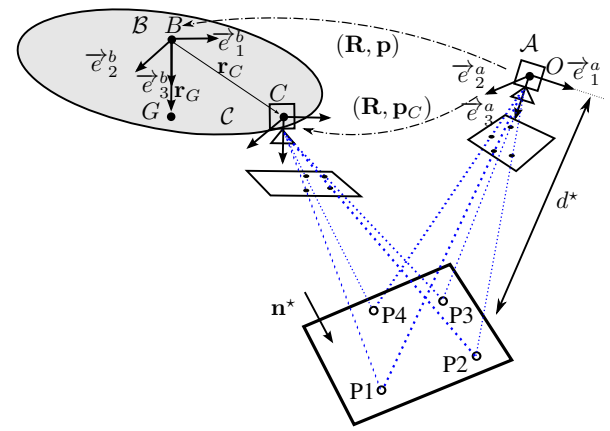

Fig. 1: An AUV with a downward-looking camera and notation

The AUV is modeled as a rigid body immersed in water. The following notation is used throughout the paper (see Fig. 1).

- Let $m$ and $\mathbf{J}_{0}$ denote respectively the vehicle's mass and inertia matrix, $G$ and $B$ its center of mass (CoM) and center of buoyancy (CoB). The distance between $G$ and $B$ is denoted as $l$. The gravity constant is denoted as $g$.

- The considered inertial frame is denoted as $\mathcal{A}=$ $\left\{O ; \vec{e}_{1}^{a}, \vec{e}_{2}^{a}, \vec{e}_{3}^{a}\right\}$. The body frame attached to the AUV with the origin at the $\mathrm{CoB}$ is denoted as $\mathcal{B}=\left\{B ; \vec{e}_{1}^{b}, \vec{e}_{2}^{b}, \vec{e}_{3}^{b}\right\}$. The camera frame with base vectors parallel to those of $\mathcal{B}$ is denoted as $\mathcal{C}=\left\{C ; \vec{e}_{1}^{c}, \vec{e}_{2}^{c}, \vec{e}_{3}^{c}\right\}$. The vectors of coordinates expressed in $\mathcal{B}$ of $\overrightarrow{B C}$ and $\overrightarrow{B G}$ are respectively denoted as $\mathbf{r}_{C} \in \mathbb{R}^{3}$ and $\mathbf{r}_{G}=l \mathbf{e}_{3} \in \mathbb{R}^{3}$, with $\mathbf{e}_{3}=[0,0,1]^{\top}$.

- The orientation (i.e. attitude) of the body frame $\mathcal{B}$ relative to the inertial frame $\mathcal{A}$ is represented by a rotation matrix $\mathbf{R} \in \mathrm{SO}(3)$. By denoting $\mathbf{p}$ and $\mathbf{p}_{C}$ the vector of coordinates expressed in $\mathcal{A}$ of $B$ and $C$ respectively, one verifies that $\mathbf{p}=\mathbf{p}_{C}-\mathbf{R r}_{C}$.
- Let $\boldsymbol{\Omega}=\left[\omega_{1}, \omega_{2}, \omega_{3}\right]^{\top} \in \mathbb{R}^{3}$ denotes the angular velocity of $\mathcal{B}$ with respect to $\mathcal{A}$, expressed in $\mathcal{B}$. By denoting $\mathbf{V} \in \mathbb{R}^{3}$ and $\mathbf{V}_{C} \in \mathbb{R}^{3}$ respectively the vector of coordinates expressed in $\mathcal{B}$ of the linear velocity of $B$ and $C$, one has $\mathbf{V}=\mathbf{V}_{C}-\boldsymbol{\Omega} \times \mathbf{r}_{C}$. - The vectors of coordinates of the current velocity expressed in $\mathcal{A}$ and $\mathcal{B}$ are denoted as $\mathbf{v}_{f}$ and $\mathbf{V}_{f}$, respectively. Then the CoB's velocity relative to the current expressed in $\mathcal{B}$ is denoted as $\mathbf{V}_{h} \triangleq \mathbf{V}-\mathbf{V}_{f}$. Assume that $\mathbf{v}_{f}$ is constant.

- Let $\left\{\mathbf{e}_{1}, \mathbf{e}_{2}, \mathbf{e}_{3}\right\}$ denote the canonical basis of $\mathbb{R}^{3}$ and $\mathbf{I}_{3}$ denote the identity of $\mathbb{R}^{3 \times 3}$. For all $\mathbf{u} \in \mathbb{R}^{3}, \mathbf{u}_{\times}$denotes the skew-symmetric matrix associated with the cross product by $\mathbf{u}$, i.e., $\mathbf{u}_{\times} \mathbf{v}=\mathbf{u} \times \mathbf{v}, \forall \mathbf{v} \in \mathbb{R}^{3}$. Let $\operatorname{vex}(\cdot)$ denote an operator such that $\operatorname{vex}\left(\mathbf{u}_{\times}\right)=\mathbf{u}$. The transpose operator is denoted as $(\cdot)^{\top}$. The Euclidean norm in $\mathbb{R}^{n}$ and the Frobenius norm in $\mathbb{R}^{n \times n}$ are denoted as $|\cdot|$ and $\|\cdot\|$, respectively. The notation $\operatorname{sat}^{\delta}(\cdot) \in \mathbb{R}^{n}$, with $\delta>0$, denotes the classical saturation function, i.e., $\operatorname{sat}^{\delta}(\mathbf{x}) \triangleq \mathbf{x} \min (1, \delta /|\mathbf{x}|), \forall \mathbf{x} \in \mathbb{R}^{n}$.

\section{B. Problem formulation}

Assume that the AUV, equipped with a downward-looking monocular camera, operates sufficiently close to a (near) planar textured seafloor. To perform the station-keeping task, a reference image of the seafloor is first taken at a desired pose. Then, the visual servoing controller must stabilize the AUV about the desired pose by exploiting information encoded in both the current and reference images.

Let us define the inertial frame $\mathcal{A}$ attached to the camera's desired pose as depicted in Fig. 1. Assume that the estimation of the so-called homography $\mathbf{H} \in \mathbb{R}^{3 \times 3}$ between the current image and the reference image is available for control design. Such a homography matrix contains coupled information about the rotation and translation between the current camera frame $\mathcal{C}$ and the inertial $\mathcal{A}$ as follows [3]

$$
\mathbf{H}=\mathbf{R}^{\top}-\frac{1}{d^{\star}} \mathbf{R}^{\top} \mathbf{p}_{C} \mathbf{n}^{\star \top}
$$

where $\mathbf{n}^{\star} \in S^{2}$ is the unit normal vector pointing toward the target plane with coordinates expressed in $\mathcal{A}$; and $d^{\star}$ is the distance between the camera optical center at the desired pose and the target plane (see Fig. 1). The derivative of $\mathbf{H}$ satisfies

$$
\dot{\mathbf{H}}=-\boldsymbol{\Omega}_{\times} \mathbf{H}-\frac{1}{d^{\star}} \mathbf{V}_{C} \mathbf{n}^{\star \top}
$$

In addition to the estimate of $\mathbf{H}$, (approximate) measurements of the angular velocity $\Omega$ and of the gravity direction $\mathbf{R}^{\top} \mathbf{e}_{3}$ provided by an IMU are also available for control design.

The considered control objective consists in asymptotically stabilize $\mathbf{H}$ about $\mathbf{I}_{3}$, which is equivalent to the stabilization of $\left(\mathbf{R}, \mathbf{p}_{C}\right)$ about $\left(\mathbf{I}_{3}, \mathbf{0}\right)$. Main difficulties for control design are related to the unknown quantities $d^{\star}$ and $\mathbf{n}^{\star}$ involved in the expression (1) of the homography $\mathbf{H}$, the coupled rotation and translation transformations encoded in $\mathbf{H}$, and last but not least the unavailability of linear velocity measurements (i.e. DVL is not used).

\section{Discussions on an existing HBVS control approach}

An advanced HBVS approach without relying on homography decomposition has been proposed in [3]. This kinematic 
control approach consists in using $\left(\mathbf{V}_{C}, \boldsymbol{\Omega}\right)$ as control inputs to stabilize the visual errors $\mathbf{e}_{p}, \mathbf{e}_{\Theta} \in \mathbb{R}^{3}$ to zero, where

$$
\mathbf{e}_{p} \triangleq\left(\mathbf{I}_{3}-\mathbf{H}\right) \mathbf{m}^{\star}, \quad \mathbf{e}_{\Theta} \triangleq \operatorname{vex}\left(\mathbf{H}^{\top}-\mathbf{H}\right)
$$

with $\mathbf{m}^{\star} \in S^{2}$ an arbitrary unit vector satisfying $\mathbf{n}^{\star \top} \mathbf{m}^{\star}>$ 0 . In the case of station keeping using a downward-looking camera, the angle between the target plane's normal vector and the camera axis at the desired pose should be smaller than 90 degree, which implies that $\mathbf{n}^{\star \top} \mathbf{e}_{3}>0$. It follows that the simple choice $\mathbf{m}^{\star}=\mathbf{e}_{3}$ ensures the validity of the condition $\mathbf{n}^{\star \top} \mathbf{m}^{\star}>0$ without any prior knowledge of $\mathbf{n}^{\star}$. This choice (i.e. $\mathbf{m}^{\star}=\mathbf{e}_{3}$ ) will be considered throughout the present paper.

The following lemma states the local exponential stability of the kinematic controller proposed in [3].

Lemma 1 (See [3]) The following kinematic controller

$$
\mathbf{V}_{C}=-k_{p} \mathbf{e}_{p}, \quad \Omega=-k_{\Theta} \mathbf{e}_{\Theta}
$$

with positive gains $k_{p}$ and $k_{\Theta}$ ensures the local exponential stability of the equilibrium $\left(\mathbf{R}, \mathbf{p}_{C}\right)=\left(\mathbf{I}_{3}, \mathbf{0}\right)$, i.e. $\mathbf{H}=\mathbf{I}_{3}$.

For AUV control design, forces and torques must be used as control inputs instead of the linear and angular velocities. However, the passage from kinematic control to dynamic control requires these velocity measurements as discussed in our prior work [17]. It is thus inapplicable to the problem here considered due to the unavailability of linear velocity measurements for control design. In the sequel, a novel dynamic HBVS control approach will be developed to address the lack of linear velocity measurements.

\section{SYSTEM MODELING}

\section{A. Recalls on system modeling}

The kinematic equations of the vehicle are given by

$$
\begin{gathered}
\dot{\mathbf{p}}=\mathbf{R V} \\
\dot{\mathbf{R}}=\mathbf{R} \boldsymbol{\Omega}_{\times}
\end{gathered}
$$

The vehicle's dynamic equations are derived based on the translational and rotational momentums of the body-fluid system. The total kinetic energy of the body-fluid system is $E_{T}=E_{B}+E_{F}$ where $E_{B}$ and $E_{F}$ are the kinetic energy of the vehicle and of the surrounding fluid, respectively:

$$
\begin{gathered}
E_{B}=\frac{1}{2} \mathbf{W}_{h}^{\top} \mathbf{M}_{B} \mathbf{W}_{h}, \text { with } \mathbf{M}_{B} \triangleq\left[\begin{array}{cc}
m \mathbf{I}_{3} & -m \mathbf{r}_{G \times} \\
m \mathbf{r}_{G \times} & \mathbf{J}_{0}
\end{array}\right] \\
E_{F}=\frac{1}{2} \mathbf{W}_{h}^{\top} \mathbf{M}_{A} \mathbf{W}_{h}, \text { with } \mathbf{M}_{A} \triangleq\left[\begin{array}{cc}
\mathbf{M}_{A}^{11} & \mathbf{M}_{A}^{12} \\
\mathbf{M}_{A}^{21} & \mathbf{M}_{A}^{22}
\end{array}\right]
\end{gathered}
$$

with $\mathbf{W}_{h} \triangleq\left[\mathbf{V}_{h}^{\top}, \boldsymbol{\Omega}^{\top}\right]^{\top} \in \mathbb{R}^{6}$, and $\mathbf{M}_{A} \in \mathbb{R}^{6 \times 6}$ the hydrodynamic added-mass matrix. One thus deduces

$$
E_{T}=\frac{1}{2} \mathbf{W}_{h}^{\top} \mathbf{M}_{T} \mathbf{W}_{h}, \text { with } \mathbf{M}_{T}=\left[\begin{array}{cc}
\mathbf{M} & \mathbf{D}^{\top} \\
\mathbf{D} & \mathbf{J}
\end{array}\right]
$$

and $\mathbf{M} \triangleq m \mathbf{I}_{3}+\mathbf{M}_{A}^{11}, \mathbf{J} \triangleq \mathbf{J}_{0}+\mathbf{M}_{A}^{22}, \mathbf{D} \triangleq m \mathbf{r}_{G \times}+\mathbf{M}_{A}^{21}$. From there, the translational and rotational momentums are computed as

$$
\left\{\begin{array}{l}
\mathbf{P}_{h}=\frac{\partial E_{T}}{\partial \mathbf{V}_{h}}=\mathbf{M} \mathbf{V}_{h}+\mathbf{D}^{\top} \boldsymbol{\Omega} \\
\boldsymbol{\Pi}_{h}=\frac{\partial E_{T}}{\partial \boldsymbol{\Omega}}=\mathbf{J} \boldsymbol{\Omega}+\mathbf{D} \mathbf{V}_{h}
\end{array}\right.
$$

Then, according to the formulation of Leonard [18], the vehicle's dynamics are given by

$$
\begin{aligned}
\dot{\mathbf{P}}_{h} & =\mathbf{P}_{h} \times \boldsymbol{\Omega}+\mathbf{F}_{c}+\mathbf{F}_{g b}+\mathbf{F}_{d} \\
\dot{\boldsymbol{\Pi}}_{h} & =\boldsymbol{\Pi}_{h} \times \boldsymbol{\Omega}+\mathbf{P}_{h} \times \mathbf{V}_{h}+\boldsymbol{\Gamma}_{c}+\boldsymbol{\Gamma}_{g}+\boldsymbol{\Gamma}_{d}
\end{aligned}
$$

where $\mathbf{F}_{c} \in \mathbb{R}^{3}$ and $\boldsymbol{\Gamma}_{c} \in \mathbb{R}^{3}$ are the control force and torque vertors, $\mathbf{F}_{g b} \triangleq\left(m g-F_{b}\right) \mathbf{R}^{\top} \mathbf{e}_{3}$ the sum of the gravity and buoyancy forces, $\boldsymbol{\Gamma}_{g} \triangleq m g l \mathbf{e}_{3} \times \mathbf{R}^{\top} \mathbf{e}_{3}$ the gravity torque, and $\mathbf{F}_{d}$ and $\boldsymbol{\Gamma}_{d}$ the damping force and torque modeled as the sum of linear and quadratic terms as follows

$$
\left\{\begin{array}{l}
\mathbf{F}_{d}\left(\mathbf{V}_{h}\right)=-\left(\mathbf{D}_{V l}+\left|\mathbf{V}_{h}\right| \mathbf{D}_{V q}\right) \mathbf{V}_{h} \\
\boldsymbol{\Gamma}_{d}(\boldsymbol{\Omega})=-\left(\mathbf{D}_{\Omega l}+|\boldsymbol{\Omega}| \mathbf{D}_{\Omega q}\right) \boldsymbol{\Omega}
\end{array}\right.
$$

with positive damping matrices $\mathbf{D}_{V l}, \mathbf{D}_{V q}, \mathbf{D}_{\Omega l}, \mathbf{D}_{\Omega q} \in \mathbb{R}^{3 \times 3}$. Note that the model $\left[8\right.$ of $\mathbf{F}_{d}$ and $\boldsymbol{\Gamma}_{d}$ is not used for control design but is only useful for simulation purposes.

\section{B. Simplified model for control design}

The translational and rotational dynamics (7a)-(7b) are tightly coupled due to the coupling matrix $\mathbf{D}$ involved in the definition (6) of the momentum terms. These complex dynamic couplings are often neglected in the literature by neglecting all terms involving $\mathbf{D}$ using the fact that the considered AUV is compact and the distance between the $\mathrm{CoB}$ and CoM is relatively small. Moreover, since the linear velocity is not measured, the "Munk moment" $\left(\mathbf{M} \mathbf{V}_{h}\right) \times \mathbf{V}_{h}$ is here considered as a disturbance. Finally, all terms involving unknown current velocity $\mathbf{V}_{f}$, together with the damping force and torque, are also considered as disturbances. These considerations result in the following simpler dynamic equations that decouple the translational and rotational dynamics:

$$
\begin{aligned}
\mathbf{M} \dot{\mathbf{V}} & =(\mathbf{M V}) \times \boldsymbol{\Omega}+\mathbf{F}_{c}+\mathbf{F}_{g b}+\boldsymbol{\Delta}_{F} \\
\mathbf{J} \dot{\boldsymbol{\Omega}} & =(\mathbf{J} \boldsymbol{\Omega}) \times \boldsymbol{\Omega}+\boldsymbol{\Gamma}_{c}+\boldsymbol{\Gamma}_{g}+\boldsymbol{\Delta}_{\Gamma}
\end{aligned}
$$

with the "disturbance" terms

$$
\begin{aligned}
& \boldsymbol{\Delta}_{F} \triangleq-(\mathbf{M V})_{\times} \boldsymbol{\Omega}-\mathbf{M} \boldsymbol{\Omega}_{\times} \mathbf{V}_{f}+\left(\mathbf{D}^{\top} \boldsymbol{\Omega}\right)_{\times} \boldsymbol{\Omega}-\mathbf{D}^{\top} \dot{\boldsymbol{\Omega}}+\mathbf{F}_{d} \\
& \boldsymbol{\Delta}_{\Gamma} \triangleq\left(\mathbf{D V} \mathbf{V}_{h}\right) \times \mathbf{\Omega}+\mathbf{P}_{h} \times \mathbf{V}_{h}-\mathbf{D} \dot{\mathbf{V}}_{h}+\boldsymbol{\Gamma}_{d}
\end{aligned}
$$

In the case of station keeping, $\boldsymbol{\Delta}_{F}$ and $\boldsymbol{\Delta}_{\Gamma}$ would eventually converge to constant vectors. In the sequel, these terms will be first neglected in the derivation of a basic controller, which later on will be robustified via both integral correction actions and a high-gain observer of $\boldsymbol{\Delta}_{\Gamma}$.

\section{CONTROL DESIGN}

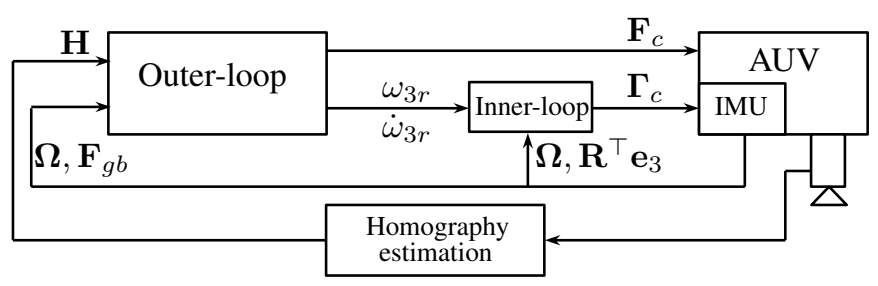

Fig. 2: Control architecture of the proposed HBVS

By analogy to the cascade inner-outer loop control architecture proposed in [17], the following modified version 
(illustrated by Fig. 2p handling the unavailability of linear velocity measurements is adopted:

- An inner-loop controller, governing the rotation dynamics (5b) and (9b), defines the torque control vector $\Gamma_{c}$ to ensure the asymptotic stabilization of $\left(\boldsymbol{\Omega}, \mathbf{R}^{\top} \mathbf{e}_{3}\right)$ about $\left(\boldsymbol{\Omega}_{r}, \mathbf{e}_{3}\right)$, where the reference angular velocity $\boldsymbol{\Omega}_{r}$ is defined by

$$
\boldsymbol{\Omega}_{r} \triangleq k_{g} \mathbf{e}_{3} \times \mathbf{R}^{\top} \mathbf{e}_{3}+\omega_{3 r} \mathbf{e}_{3}
$$

with $k_{g}>0$ positive gain and $\omega_{3 r} \in \mathbb{R}$ the reference yaw angular velocity to be specified by the outer-loop controller. Note that $\dot{\omega}_{3 r}$ must be computable so that the feedforward term $\dot{\Omega}_{r}$ is also computable by the torque controller.

- An outer-loop controller defines the force control vector $\mathbf{F}_{c}$ together with the reference yaw angular velocity $\omega_{3 r}$ (for the inner-loop controller) to fulfill the main objective of stabilizing $\mathbf{H}$ about $\mathbf{I}_{3}$, provided that the inner-loop controller ensures the almost global asymptotic stability (almost-GAS) and local exponential stability (LES) of the equilibrium $\left(\boldsymbol{\Omega}, \mathbf{R}^{\top} \mathbf{e}_{3}\right)=\left(\boldsymbol{\Omega}_{r}, \mathbf{e}_{3}\right)$.

The inner-loop control design is less involved and is postponed after the outer-loop control design, which is the main contribution of this paper.

\section{A. Outer-loop control design}

1) Force control design: Analogously to our prior work [17], the control design for stabilizing the visual error $\mathbf{e}_{p}$ defined in (3) about zero is first carried out, but here without linear velocity measurements.

In view of (2) and (3) and using the choice $\mathbf{m}^{\star}=\mathbf{e}_{3}$, the dynamics of $\mathbf{e}_{p}$ satisfy

$$
\dot{\mathbf{e}}_{p}=-\boldsymbol{\Omega} \times\left(\mathbf{e}_{p}-\mathbf{e}_{3}\right)+a^{\star} \mathbf{V}_{C}
$$

with $a^{\star} \triangleq \frac{\mathbf{n}^{\star \top} \mathbf{e}_{3}}{d^{\star}}$ an unknown number. However, we know that it is positive using the condition $\mathbf{n}^{\star \top} \mathbf{e}_{3}>0$.

In our prior work [27] the outer-loop controller has been designed based on the assumption that the vehicle's $\mathrm{CoB} B$ and CoM $G$ and the camera optical center $C$ are aligned. This restrictive assumption is not used in the present paper. To this purpose let us introduce a modified velocity variable

$$
\overline{\mathbf{V}} \triangleq \mathbf{V}+\omega_{3 r} \mathbf{e}_{3} \times \mathbf{r}_{C}
$$

As a result of the inner-loop controller (to be designed thereafter) that ensures the exponential convergence of $\left(\Omega, \mathbf{R}^{\top} \mathbf{e}_{3}\right)$ to $\left(\boldsymbol{\Omega}_{r}, \mathbf{e}_{3}\right)$, one ensures that $\boldsymbol{\Omega}$ exponentially converges to $\omega_{3 r} \mathbf{e}_{3}$, which in turn implies that $\mathbf{V}_{C}$ exponentially converges to $\overline{\mathbf{V}}$ and $\boldsymbol{\Omega} \times \mathbf{e}_{3}$ exponentially converges to zero. Therefore, (11) can be rewritten as

$$
\dot{\mathbf{e}}_{p}=-\boldsymbol{\Omega} \times \mathbf{e}_{p}+a^{\star} \overline{\mathbf{V}}+\boldsymbol{\varepsilon}(t)
$$

with exponentially vanishing term

$$
\varepsilon(t) \triangleq \boldsymbol{\Omega} \times \mathbf{e}_{3}+a^{\star}\left(\boldsymbol{\Omega}-\boldsymbol{\Omega}_{r}\right) \times \mathbf{r}_{C}
$$

On the other hand, using $9 \mathrm{a}$ the dynamics of $\overline{\mathbf{V}}$ verify

$$
\begin{aligned}
\mathbf{M} \dot{\overline{\mathbf{V}}}= & (\mathbf{M} \overline{\mathbf{V}}) \times \boldsymbol{\Omega}+\mathbf{F}_{c}+\mathbf{F}_{g b}+\boldsymbol{\Delta}_{F} \\
& +\dot{\omega}_{3 r} \mathbf{M}\left(\mathbf{e}_{3} \times \mathbf{r}_{C}\right)-\omega_{3 r}\left(\mathbf{M}\left(\mathbf{e}_{3} \times \mathbf{r}_{C}\right)\right) \times \mathbf{\Omega} \\
= & (\mathbf{M} \overline{\mathbf{V}}) \times \boldsymbol{\Omega}+\overline{\mathbf{F}}_{c}+\mathbf{F}_{g b}+\boldsymbol{\Delta}_{F}+\boldsymbol{\varepsilon}_{1}(t)
\end{aligned}
$$

with new control force variable

$$
\begin{aligned}
\overline{\mathbf{F}}_{c} & \triangleq \mathbf{F}_{c}+\dot{\omega}_{3 r} \mathbf{M}\left(\mathbf{e}_{3} \times \mathbf{r}_{C}\right)-\omega_{3 r}^{2}\left(\mathbf{M}\left(\mathbf{e}_{3} \times \mathbf{r}_{C}\right)\right) \times \mathbf{e}_{3} \\
& =\mathbf{F}_{c}+\left(\dot{\omega}_{3 r} \mathbf{I}_{3}+\omega_{3 r}^{2}\left[\mathbf{e}_{3}\right]_{\times}\right) \mathbf{M}\left(\mathbf{e}_{3} \times \mathbf{r}_{C}\right)
\end{aligned}
$$

and exponentially vanishing term

$$
\varepsilon_{1}(t) \triangleq-\omega_{3 r}\left(\mathbf{M}\left(\mathbf{e}_{3} \times \mathbf{r}_{C}\right)\right) \times\left(\boldsymbol{\Omega}-\boldsymbol{\Omega}_{r}\right)
$$

To expose the main ideas of control design, the outer-loop control design will be first carried out for the case where the disturbance term $\Delta_{F}$ involved in 15 is considered null, i.e. $\boldsymbol{\Delta}_{F} \equiv \mathbf{0}$. Then, we will show later on how to cope with external disturbances and model uncertainties via integral correction actions.

Proposition 1 Consider system including the dynamics (13) of $\mathbf{e}_{p}$ and translation dynamics given by $\left[15\right.$ with $\boldsymbol{\Delta}_{F} \equiv \mathbf{0}$. Assume that $\Omega$ remains bounded for all time. Assume that the disturbance terms $\varepsilon(t)$ and $\varepsilon_{1}(t)$ remain also bounded for all time and converge asymptotically to zero and with exponential rate of convergence after some time instant $T_{\varepsilon} \geq 0$. Introduce the augmented system

$$
\dot{\hat{\mathbf{e}}}_{p}=-\boldsymbol{\Omega} \times \hat{\mathbf{e}}_{p}-\mathbf{K}_{1} \hat{\mathbf{e}}_{p}+\mathbf{K}_{1} \mathbf{e}_{p}, \quad \hat{\mathbf{e}}_{p}(0) \in \mathbb{R}^{3}
$$

with $\mathbf{K}_{1} \in \mathbb{R}^{3 \times 3}$ positive definite gain matrix. Apply the control force

$$
\overline{\mathbf{F}}_{c}=\bar{m} \mathbf{M}^{-1}\left(k_{2} \hat{\mathbf{e}}_{p}-\left(k_{2}+k_{3}\right) \mathbf{e}_{p}\right)-\mathbf{F}_{g b}
$$

with $\bar{m}, k_{2}, k_{3}>0$. Then, the equilibrium $\left(\hat{\mathbf{e}}_{p}, \mathbf{e}_{p}, \overline{\mathbf{V}}\right)=$ $(\mathbf{0}, \mathbf{0}, \mathbf{0})$ is globally asymptotically stable $(G A S)$.

Proof: Define $\tilde{\mathbf{e}}_{p} \triangleq \hat{\mathbf{e}}_{p}-\mathbf{e}_{p}$. From (13) and (18) one obtains

$$
\dot{\tilde{\mathbf{e}}}_{p}=-\boldsymbol{\Omega} \times \tilde{\mathbf{e}}_{p}-\mathbf{K}_{1} \tilde{\mathbf{e}}_{p}-a^{\star} \overline{\mathbf{V}}-\boldsymbol{\varepsilon}(t)
$$

Define $\mathbf{x}_{1} \triangleq\left[\tilde{\mathbf{e}}_{p}^{\top}, \mathbf{e}_{p}^{\top}, \mathbf{M} \overline{\mathbf{V}}^{\top}\right]^{\top}, \mathbf{x}_{2} \triangleq\left[\varepsilon^{\top}, \boldsymbol{\varepsilon}_{1}^{\top}\right]^{\top}$. One verifies from (14) and (17) that $\mathrm{x}_{2}$ is independent with $\mathrm{x}_{1}$. Thus, the closed-loop system of system $(13)+15+20$ can be rewritten in the following cascaded time-varying system studied by Panteley and Loria [29

$$
\dot{\mathbf{x}}_{1}=\mathbf{A}(t) \mathbf{x}_{1}+\mathbf{B} \mathbf{x}_{2}
$$

with

$$
\mathbf{A}(t) \triangleq\left[\begin{array}{ccc}
-\boldsymbol{\Omega}_{\times}-\mathbf{K}_{1} & \mathbf{0} & -a^{\star} \mathbf{M}^{-1} \\
\mathbf{0} & -\boldsymbol{\Omega}_{\times} & a^{\star} \mathbf{M}^{-1} \\
k_{2} \bar{m} \mathbf{M}^{-1} & -k_{3} \bar{m} \mathbf{M}^{-1} & -\boldsymbol{\Omega}_{\times}
\end{array}\right]
$$

and $\mathbf{B}$ a constant matrix composed of 0 and 1 . System 21, can be seen as a nominal system

$$
\dot{\mathbf{x}}_{1}=\mathbf{A}(t) \mathbf{x}_{1}
$$

perturbed by the output $\mathbf{x}_{2}$ of a stable system that remains bounded for all time and converges to zero with exponential rate when $t \geq T_{\varepsilon}$.

To apply [29, Theorem 2] one needs to verify first that the state $\mathbf{x}_{1}$ of the nominal system (22) remains bounded in the

\footnotetext{
${ }^{1}$ The class of cascade time-varying systems studied by Panteley and Loria is of the form: $\dot{\mathbf{x}}_{1}=\mathbf{f}\left(t, \mathbf{x}_{1}\right)+\mathbf{g}\left(t, \mathbf{x}_{1}, \mathbf{x}_{2}\right) \mathbf{x}_{2}$. Thus, in our case $\mathbf{f}\left(t, \mathbf{x}_{1}\right)=$ $\mathbf{A}(t) \mathbf{x}_{1}$ and $\mathbf{g}\left(t, \mathbf{x}_{1}, \mathbf{x}_{2}\right)=\mathbf{B}$.
} 
time interval $\left[0, T_{\varepsilon}\right]$. Indeed, since $\Omega, \varepsilon$ and $\varepsilon_{1}$ are bounded for all time (and subsequently in $\left[0, T_{\varepsilon}\right]$ ) by assumption there exist two positive numbers $\beta_{1}, \beta_{2}>0$ such that

$$
\left|\dot{\mathbf{x}}_{1}(t)\right| \leq \beta_{1}\left|\mathbf{x}_{1}(t)\right|+\beta_{2}, \quad \forall t \in\left[0, T_{\varepsilon}\right]
$$

This inequality implies that the solutions $\mathbf{x}_{1}$ cannot grow faster than exponentially and, thus, do not blow up in finite time. From there, one can conveniently consider System (21) with initial time $t_{0}=T_{\varepsilon}$ and apply [29. Theorem 2] to this system. The next step consists in verifying the validation of all assumptions of that theorem.

One of the main requirements of [29. Theorem 2] is that the origin of the nominal system (22) is globally uniformly asymptotically stable. Consider the following Lyapunov function candidate

$$
\mathcal{L} \triangleq \frac{k_{2}}{2}\left|\tilde{\mathbf{e}}_{p}\right|^{2}+\frac{k_{3}}{2}\left|\mathbf{e}_{p}\right|^{2}+\frac{a^{\star}}{2 \bar{m}}|\mathbf{M} \overline{\mathbf{V}}|^{2}
$$

One verifies that the time-derivative of $\mathcal{L}$ along any solution to the controlled unperturbed system 22, is

$$
\begin{aligned}
\dot{\mathcal{L}}= & -k_{2} \tilde{\mathbf{e}}_{p}^{\top} \mathbf{K}_{1} \tilde{\mathbf{e}}_{p}-a^{\star} k_{2} \tilde{\mathbf{e}}_{p}^{\top} \overline{\mathbf{V}}+a^{\star} k_{3} \mathbf{e}_{p}^{\top} \overline{\mathbf{V}} \\
& +a^{\star} \bar{m}^{-1}(\mathbf{M} \overline{\mathbf{V}})^{\top}\left(\overline{\mathbf{F}}_{c}+\mathbf{F}_{g b}\right) \\
= & -k_{2} \tilde{\mathbf{e}}_{p}^{\top} \mathbf{K}_{1} \tilde{\mathbf{e}}_{p}
\end{aligned}
$$

From (24) one deduces that $\dot{\mathcal{L}}$ is semi-negative definite and, thus, $\tilde{\mathbf{e}}_{p}, \mathbf{e}_{p}$, and $\overline{\mathbf{V}}$ remain bounded by initial conditions. One then easily verifies that $\dot{\tilde{\mathbf{e}}}_{p}$ and $\ddot{\mathcal{L}}$ also remain bounded, implying the uniform continuity of $\dot{\mathcal{L}}$. Then, application of Barbalat's lemma [16] ensures the convergence of $\dot{\mathcal{L}}$ and, thus, of $\tilde{\mathbf{e}}_{p}$ to zero. Then, from 20 (with $\varepsilon(t) \equiv \mathbf{0}$ ) and by application of the extended Barbalat's lemma [26] one also ensures the convergence of $\dot{\tilde{\mathbf{e}}}_{p}$ to zero, which in turn implies that $\overline{\mathbf{V}}$ converges to zero. Similar arguments can be used to show the convergence of $\dot{\overline{\mathbf{V}}}$ to zero. In view of (15) (with $\left.\boldsymbol{\Delta}_{F} \equiv \mathbf{0}\right), \varepsilon_{1}(t) \equiv \mathbf{0}$ and $\overline{\mathbf{F}}_{c}$ given by (19), one ensures that $\mathbf{e}_{p}$ converges to zero. The stability of the equilibrium $\left(\mathbf{e}_{p}, \hat{\mathbf{e}}_{p}, \overline{\mathbf{V}}\right)=(\mathbf{0}, \mathbf{0}, \mathbf{0})$ (or $\left.\mathbf{x}_{1}=\mathbf{0}\right)$ is a direct consequence of (23) and (24).

We have proved that the origin of the nominal system (22) is GAS. The next step consists in showing [29, inequality (7)] is satisfied with the Lyapunov function 23 and for the nominal system (22). This condition consists in proving the existence of $c_{1}>0, \delta_{1} \geq 0$ such that

$$
\left|\frac{\partial \mathcal{L}}{\partial \mathbf{x}_{1}}\right|\left|\mathbf{x}_{1}\right| \leq c_{1} \mathcal{L}, \forall\left|\mathbf{x}_{1}\right| \geq \delta_{1}
$$

From the definition 24 of $\mathcal{L}$ one deduces

$$
\alpha_{1}\left|\mathbf{x}_{1}\right|^{2} \leq \mathcal{L} \leq \alpha_{2}\left|\mathbf{x}_{1}\right|^{2}
$$

with $\alpha_{1} \triangleq \min \left(\frac{k_{2}}{2}, \frac{k_{3}}{2}, \frac{a^{\star}}{2 \bar{m}}\right), \alpha_{2} \triangleq \max \left(\frac{k_{2}}{2}, \frac{k_{3}}{2}, \frac{a^{\star}}{2 \bar{m}}\right)$. One also verifies that $\left|\frac{\partial \mathcal{L}}{\partial \mathbf{x}_{1}}\right| \leq 2 \sqrt{3} \alpha_{2}\left|\mathbf{x}_{1}\right|$. Thus, inequality 25 is satisfied with $c_{1}=2 \sqrt{3} \alpha_{2} / \alpha_{1}$ and $\delta_{1}=0$.

It remains to verify Assumptions A2 and A3 of [29. Theorem 2]. Indeed, Assumption A2 on the interconnection term $\mathbf{B}$ is clearly satisfied since $\mathbf{B}$ is constant (i.e. $\exists c_{\mathbf{B}}>0$ : $|\mathbf{B}| \leq c_{\mathbf{B}}$ ) which implies that [29, inequality (9)] is satisfied, i.e.

$$
\left|\mathbf{B}\left(t, \mathbf{x}_{1}, \mathbf{x}_{2}\right)\right| \leq \theta_{1}\left(t,\left|\mathbf{x}_{2}\right|\right)+\theta_{2}\left(t,\left|\mathbf{x}_{2}\right|\right)\left|\mathbf{x}_{1}\right|
$$

with $\theta_{1}\left(t,\left|\mathbf{x}_{2}\right|\right)=c_{\mathbf{B}}$ and $\theta_{2}\left(t,\left|\mathbf{x}_{2}\right|\right)=0$.

One can verify that Assumption A3 is also satisfied using the fact that $\left|\mathbf{x}_{2}\left(t \geq t_{0}\right)\right|$ exponentially converges to zero (with initial time $t_{0}=T_{\varepsilon}$ as explained previously). Indeed, there exist $\gamma_{1}, \gamma_{2}>0$ such that

$$
\left|\mathbf{x}_{2}\left(t, t_{0}, \mathbf{x}_{2}\left(t_{0}\right)\right)\right| \leq \gamma_{1}\left|\mathbf{x}_{2}\left(t_{0}\right)\right| e^{-\gamma_{2}\left(t-t_{0}\right)}, \forall t \geq t_{0}
$$

and, subsequently, 29, inequality (10)] holds, that is

$$
\int_{t_{0}}^{\infty}\left|\mathbf{x}_{2}\left(t, t_{0}, \mathbf{x}_{2}\left(t_{0}\right)\right)\right| d t \leq \phi\left(\left|\mathbf{x}_{2}\left(t_{0}\right)\right|\right) \triangleq \frac{\gamma_{1}}{\gamma_{2}}\left|\mathbf{x}_{2}\left(t_{0}\right)\right|
$$

with $\phi(\cdot)$ a class $\mathcal{K}$ function (i.e. strictly increasing and vanishing at zero). From there, the application of [29. Theorem 2] ensures that the equilibrium $\left(\mathbf{x}_{1}, \mathbf{x}_{2}\right)=(\mathbf{0}, \mathbf{0})$ is GAS. This concludes the proof.

Note that Proposition 1 applies to the case where the perturbation term $\Delta_{F}$ is negligible. While this basic controller would be able to handle small currents, in practice it is often desirable to enhance control robustness by incorporating integral correction actions. However, in our case the system considered in Proposition 1 (i.e. $[18+13+15)$ ) is already a third-order time-varying system. Thus, adding an integrator would lead to a fourth-order time-varying system. Too high order system, together with the presence of an unknown multiplicative factor $a^{\star}$ in $(13)$ and the unavailability of linear velocity measurements, excludes the possibility of establishing global (or semi-global) stability results similar to Proposition 1. However, it is still possible to state local exponential stability. For simplicity, let us consider the case where $\mathbf{M}$ can be roughly approximated by a positive diagonal matrix, i.e. $\mathbf{M} \approx \operatorname{diag}\left(m_{1,1}, m_{2,2}, m_{3,3}\right)$.

Proposition 2 Consider system including dynamics of $\mathbf{e}_{p}$ given by (13) and translation dynamics given by (15) with constant disturbance $\boldsymbol{\Delta}_{F}$ and diagonal total mass matrix $\mathbf{M}=\operatorname{diag}\left(m_{1,1}, m_{2,2}, m_{3,3}\right)$. Assume that the disturbance terms $\varepsilon(t)$ and $\varepsilon_{1}(t)$ remaining bounded for all time and converge asymptotically to zero and with exponential rate of convergence after some time instant $T_{\varepsilon}$. Introduce the following integrator

$$
\dot{\mathbf{z}}=-\boldsymbol{\Omega} \times \mathbf{z}+\mathbf{e}_{p}, \quad \mathbf{z}(0) \in \mathbb{R}^{3}
$$

and the following augmented system

$$
\dot{\hat{\mathbf{e}}}_{p}=-\boldsymbol{\Omega} \times \hat{\mathbf{e}}_{p}-\mathbf{K}_{1} \hat{\mathbf{e}}_{p}+\mathbf{K}_{1} \overline{\mathbf{e}}_{p}, \quad \hat{\mathbf{e}}_{p}(0) \in \mathbb{R}^{3}
$$

with positive diagonal matrix $\mathbf{K}_{1} \in \mathbb{R}^{3 \times 3}$ and $\overline{\mathbf{e}}_{p} \triangleq \mathbf{e}_{p}+k_{I} \mathbf{z}$ with positive integral gain $k_{I}$. Apply the control force

$$
\overline{\mathbf{F}}_{c}=\bar{m} \mathbf{M}^{-1}\left(\operatorname{sat}^{\eta_{1}}\left(k_{2} \tilde{\overline{\mathbf{e}}}_{p}\right)-\operatorname{sat}^{\eta_{2}}\left(k_{3} \overline{\mathbf{e}}_{p}\right)\right)-\mathbf{F}_{g b}
$$

with positive numbers $\bar{m}, k_{2}, k_{3}, \eta_{1}, \eta_{2}$, and $\tilde{\overline{\mathbf{e}}}_{p} \triangleq \hat{\mathbf{e}}_{p}-\overline{\mathbf{e}}_{p}$. Choose $\eta_{2}$ high enough such that

$$
\eta_{2}>\bar{m}^{-1}\left|\mathbf{M} \boldsymbol{\Delta}_{F}\right|
$$

and choose $k_{I}$ satisfying

$$
k_{I}<\frac{k_{2} \underline{\lambda}_{\mathbf{K}_{1}}}{k_{2}+k_{3}}
$$


with $\underline{\lambda}_{\mathbf{K}_{1}}$ the smallest diagonal component of $\mathbf{K}_{1}$. Assume that the outer-loop control $\omega_{3 r}$ together with the innerloop control $\boldsymbol{\Gamma}_{c}$ ensures that $\boldsymbol{\Omega}$ can be considered as a first order term in first order approximations. Then, the equilibrium $\left(\hat{\mathbf{e}}_{p}, \mathbf{e}_{p}, \overline{\mathbf{V}}, \mathbf{z}\right)=\left(k_{I} \mathbf{z}^{\star}, \mathbf{0}, \mathbf{0}, \mathbf{z}^{\star}\right)$, with $\mathbf{z}^{\star} \triangleq$ $\left(\bar{m} k_{3} k_{I}\right)^{-1} \mathbf{M} \boldsymbol{\Delta}_{F}$, of the controlled system is locally exponentially stable (LES). Moreover, $\overline{\mathbf{F}}_{c}$ remains bounded by

$$
\left|\overline{\mathbf{F}}_{c}\right| \leq \bar{m} \underline{\lambda}_{\mathbf{M}}^{-1}\left(\eta_{1}+\eta_{2}\right)+\left|m g-F_{b}\right|
$$

with $\underline{\lambda}_{\mathbf{M}}$ the smallest eigenvalue of $\mathbf{M}$.

Proof: One verifies that the linearized system of Eqs. (13), 15, 27) augmented with integrator 26 around the equilibrium $\left(\hat{\mathbf{e}}_{p}, \mathbf{e}_{p}, \overline{\mathbf{V}}, \mathbf{z}\right)=\left(k_{I} \mathbf{z}^{\star}, \mathbf{0}, \mathbf{0}, \mathbf{z}^{\star}\right)$, with $\mathbf{z}^{\star} \triangleq$ $\left(\bar{m} k_{3} k_{I}\right)^{-1} \mathbf{M} \boldsymbol{\Delta}_{F}$, is given by

$$
\dot{\mathbf{x}}_{1}=\mathbf{A} \mathbf{x}_{1}+\mathbf{B} \mathbf{x}_{2}
$$

with

$$
\begin{aligned}
\mathbf{x}_{1} \triangleq\left[\left(\hat{\mathbf{e}}_{p}-k_{I} \mathbf{z}^{\star}\right)^{\top}, \mathbf{e}_{p}^{\top},(\mathbf{M} \overline{\mathbf{V}})^{\top},\left(\mathbf{z}-\mathbf{z}^{\star}\right)^{\top}\right]^{\top} \in \mathbb{R}^{12} \\
\mathbf{x}_{2} \triangleq\left[\boldsymbol{\varepsilon}^{\top}, \boldsymbol{\varepsilon}_{1}^{\top}\right]^{\top} \in \mathbb{R}^{6} \\
\mathbf{A} \triangleq\left[\begin{array}{cccc}
-\mathbf{K}_{1} & \mathbf{K}_{1} & \mathbf{0} & k_{I} \mathbf{K}_{1} \\
\mathbf{0} & \mathbf{0} & a^{\star} \mathbf{M}^{-1} & \mathbf{0} \\
k_{2} \bar{m} \mathbf{M}^{-1} & -\left(k_{2}+k_{3}\right) \bar{m} \mathbf{M}^{-1} & \mathbf{0} & -k_{I}\left(k_{2}+k_{3}\right) \bar{m} \mathbf{M}^{-1} \\
\mathbf{0} & \mathbf{I}_{3} & \mathbf{0} & \mathbf{0}
\end{array}\right]
\end{aligned}
$$

and $\mathbf{B} \in \mathbb{R}^{12 \times 6}$ a constant matrix composed of 0 and 1 .

The 12th-order characteristic polynomial of the nominal autonomous system $\dot{\mathbf{x}}_{1}=\mathbf{A} \mathbf{x}_{1}$ is $Q(\lambda)=Q_{1}(\lambda) Q_{2}(\lambda) Q_{3}(\lambda)$, with

$$
\begin{aligned}
Q_{i}(\lambda) & =\lambda^{4}+k_{1 i} \lambda^{3}+\left(k_{2}+k_{3}\right) a^{\star} \frac{\bar{m}}{m_{i, i}^{2}} \lambda^{2} \\
& +\left(k_{1 i} k_{3}+\left(k_{2}+k_{3}\right) k_{I}\right) a^{\star} \frac{\bar{m}}{m_{i, i}^{2}} \lambda+k_{1 i} k_{3} k_{I} a^{\star} \frac{\bar{m}}{m_{i, i}^{2}}
\end{aligned}
$$

with $k_{I}$ satisfying (30). Application of Routh-Hurwitz criterion ensures the exponential stability of the nominal autonomous system. Since $\mathbf{x}_{2}$ is exponentially stable by assumption, one deduces that the cascade (32) is also exponentially stable.

One can verify that condition (29) is necessary so that the integral action can compensate for the disturbance $\boldsymbol{\Delta}_{F}$. Finally, the bound of the force control vector $\overline{\mathbf{F}}_{c}$ given by (31) is directly deduced from 28,.

Remark 1 In first order approximations, the force control $\overline{\mathbf{F}}_{c}$ given by (28) is given by

$$
\overline{\mathbf{F}}_{c}=k_{2} \bar{m} \mathbf{M}^{-1} \hat{\mathbf{e}}_{p}-\left(k_{2}+k_{3}\right) \bar{m} \mathbf{M}^{-1} \overline{\mathbf{e}}_{p}-\mathbf{F}_{g b}
$$

The proof of global asymptotic stability of the equilibrium $\left(\hat{\mathbf{e}}_{p}, \mathbf{e}_{p}, \overline{\mathbf{V}}, \mathbf{z}\right)=\left(k_{I} \mathbf{z}^{\star}, \mathbf{0}, \mathbf{0}, \mathbf{z}^{\star}\right)$, with $\overline{\mathbf{F}}_{c}$ given by (33), proceeds analogously to the proof of Proposition 2 . The linear approximation (33) of $\overline{\mathbf{F}}_{c}$ is useful for gain tuning using, for instance, pole placement technique, while its nonlinear expression (28) that involves saturation functions allows us to define explicitly the bound of the force control input $\overline{\mathbf{F}}_{c}$ as given by (31). The latter property is of particular importance in practice since it is often desirable to take explicitly actuation limitations into account. For instance, (31) implies that the desired bound of $\left|\overline{\mathbf{F}}_{c}\right|$ can be set to any value $\mu\left(>\left|m g-F_{b}\right|\right)$ if $\eta_{1}+\eta_{2} \leq \bar{m}^{-1} \underline{\lambda}_{\mathbf{M}}\left(\mu-\left|m g-F_{b}\right|\right)$.
2) Control design of the reference yaw angular velocity $\omega_{3 r}$ : The previous part of outer-loop control design ensures the convergence of $\mathbf{e}_{p}$ to zero, which in turn ensures the convergence of $\mathbf{p}_{C}$ to zero. Then, using the fact that the innerloop torque controller ensures the convergence of $\mathbf{R}^{\top} \mathbf{e}_{3}$ to $\mathbf{e}_{3}$, one easily verifies that the element $h_{1,2}$ at the first row and second column of $\mathbf{H}$ converges to $\sin \psi$, with $\psi$ to yaw Euler angle. From there, the design of $\omega_{3 r}$ can proceed identically to our prior work [17] and is, thus, recalled here for the sake of completeness.

Proposition 3 (see [17] for proof) Assume that the inner-loop torque controller $\Gamma_{c}$ ensures the almost-GAS and LES of the equilibrium $\left(\boldsymbol{\Omega}, \mathbf{R}^{\top} \mathbf{e}_{3}\right)=\left(\boldsymbol{\Omega}_{r}, \mathbf{e}_{3}\right)$, with $\boldsymbol{\Omega}_{r}$ defined by 10 ) combined with $\omega_{3 r}$ (involved in $(10)$ ) solution to the following system

$$
\dot{\omega}_{3 r}=-k_{\Theta 2} \omega_{3 r}-k_{\Theta 1} \mathrm{sat}^{\Delta_{\Theta}}\left(h_{1,2}\right), \quad \omega_{3 r}(0) \in \mathbb{R}
$$

with $k_{\Theta 1}, k_{\Theta 2}, \Delta_{\Theta}>0$ and $h_{1,2}$ the element at the first row and second column of $\mathbf{H}$. Apply the outer-loop force controller $\mathbf{F}_{c}$ given either by Proposition 1 (when $\boldsymbol{\Delta}_{F} \equiv \mathbf{0}$ ) or Proposition 2 (when $\boldsymbol{\Delta}_{F}$ is constant). Then, the equilibrium $\mathbf{H}=\mathbf{I}_{3}$ is LES. Moreover, this equilibrium is almost-GAS in the case where $\overline{\mathbf{F}}_{c}$ is given by Proposition 1 and $\boldsymbol{\Delta}_{F} \equiv \mathbf{0}$.

\section{B. Inner-loop control design}

The more involved part concerning the outer-loop control design has been presented. It remains to design an effective inner-loop torque controller that ensures the stability of the equilibrium $\left(\boldsymbol{\Omega}, \mathbf{R}^{\top} \mathbf{e}_{3}\right)=\left(\boldsymbol{\Omega}_{r}, \mathbf{e}_{3}\right)$, with $\boldsymbol{\Omega}_{r}$ defined by 10 ) combined with 34 .

In view of the rotation dynamics (i.e. (5b) and (9b), it is not too difficult to carry out the above-mentioned objective since the sub-system under consideration is fully-actuated and the measurements of both $\boldsymbol{\Omega}$ and $\mathbf{R}^{\top} \mathbf{e}_{3}$ are at our disposal. However, the troublesome term $\Delta_{\Gamma}$ involved in (9b) should be carefully addressed, especially when the vehicle is subjected to strong currents that excite the Munk moment effects. Since the angular velocity can be measured at high frequency and with good precision, we propose to estimate $\boldsymbol{\Delta}_{\Gamma}$ using a high-gain observer similarly to the idea proposed in [13].

Lemma 2 Consider the following observer of $\Delta_{\Gamma}$ :

$$
\left\{\begin{array}{l}
\mathbf{J} \dot{\hat{\mathbf{\Omega}}}=(\mathbf{J} \boldsymbol{\Omega}) \times \hat{\boldsymbol{\Omega}}+\boldsymbol{\Gamma}_{c}+\boldsymbol{\Gamma}_{g}+\hat{\boldsymbol{\Delta}}_{\Gamma}+k_{0} \mathbf{J}(\boldsymbol{\Omega}-\hat{\boldsymbol{\Omega}}) \\
\dot{\hat{\boldsymbol{\Delta}}}_{\Gamma}=a_{0}^{2} k_{0}^{2} \mathbf{J}(\boldsymbol{\Omega}-\hat{\boldsymbol{\Omega}})
\end{array}\right.
$$

with $\hat{\boldsymbol{\Omega}}$ and $\hat{\boldsymbol{\Delta}}_{\Gamma}$ the estimates of $\boldsymbol{\Omega}$ and $\boldsymbol{\Delta}_{\Gamma}$, respectively; $\hat{\boldsymbol{\Omega}}(0) \in \mathbb{R}^{3}, \hat{\boldsymbol{\Delta}}_{\Gamma}(0) \in \mathbb{R}^{3} ; a_{0}, k_{0}$ some positive gains. Assume that $\dot{\Delta}_{\Gamma}$ is uniformly ultimately bounded (u.u.b.). Then for any $a_{0} \in(1-\sqrt{2} / 2,1+\sqrt{2} / 2)$,

1) The errors $\hat{\boldsymbol{\Omega}}-\boldsymbol{\Omega}$ and $\hat{\boldsymbol{\Delta}}_{\Gamma}-\boldsymbol{\Delta}_{\Gamma}$ are u.u.b. by a positive constant $\varepsilon\left(k_{0}\right)$ that tends to zero when $k_{0}$ tends to $+\infty$. Moreover, these terms converge exponentially to zero for any $k_{0}>0$ if $\boldsymbol{\Delta}_{\Gamma}$ is constant.

2) $\dot{\hat{\Delta}}_{\Gamma}$ is u.u.b. by a constant independent of $k_{0}$. 
The proof proceeds identically to the proof of [12, Proposition 8]. Now, we can use the estimate $\hat{\boldsymbol{\Delta}}_{\Gamma}$ as a feedforward term for the inner-loop torque control design.

Define the angular velocity error variable $\tilde{\Omega} \triangleq \Omega-\boldsymbol{\Omega}_{r}$. From (9b), one obtains the following error equation

$$
\mathbf{J} \dot{\tilde{\mathbf{\Omega}}}=(\mathbf{J} \boldsymbol{\Omega})_{\times} \tilde{\boldsymbol{\Omega}}+\boldsymbol{\Gamma}_{c}+\boldsymbol{\Gamma}_{g}+\boldsymbol{\Gamma}+\hat{\boldsymbol{\Delta}}_{\Gamma}+\overline{\boldsymbol{\Delta}}_{\Gamma}
$$

with $\boldsymbol{\Gamma} \triangleq(\mathbf{J} \boldsymbol{\Omega})_{\times} \boldsymbol{\Omega}_{r}-\mathbf{J} \dot{\boldsymbol{\Omega}}_{r}$ and $\overline{\boldsymbol{\Delta}}_{\Gamma} \triangleq \boldsymbol{\Delta}_{\Gamma}-\hat{\boldsymbol{\Delta}}_{\Gamma}$.

Proposition 4 Consider error equation given by (36). Assume that the unknown perturbation term $\overline{\boldsymbol{\Delta}}_{\Gamma}$ is constant and bounded by a known value $\bar{\epsilon}$. Define an anti-windup integrator $\mathbf{I}_{\Omega}$ solution to the following differential equation

$$
\dot{\mathbf{I}}_{\Omega}=-k \mathbf{I}_{\Omega}+\operatorname{sat}^{\Delta_{1}}\left(k \mathbf{I}_{\Omega}+\operatorname{sat}^{\Delta_{2}}(\mathbf{Q} \tilde{\boldsymbol{\Omega}})\right), \mathbf{I}_{\Omega}(0) \in \mathbb{R}^{3}
$$

with $k$ a positive gain, $\Delta_{1}, \Delta_{2}$ some positive constants, and $\mathbf{Q} \in \mathbb{R}^{3 \times 3}$ satisfying $\mathbf{Q}^{\top} \mathbf{Q}=\mathbf{J}$. Apply the control torque

$$
\begin{aligned}
\boldsymbol{\Gamma}_{c}= & -\operatorname{sat}^{\eta_{3}}\left(\mathbf{K}_{\Omega} \tilde{\boldsymbol{\Omega}}\right)-k_{i \Omega} \mathbf{Q}^{\top} \mathbf{I}_{\Omega} \\
& +\max \left(0, \frac{|\mathbf{Q} \tilde{\boldsymbol{\Omega}}|}{\Delta_{2}}-1\right) \boldsymbol{\Gamma}_{g}-\boldsymbol{\Gamma}-\hat{\boldsymbol{\Delta}}_{\Gamma}
\end{aligned}
$$

with $\mathbf{K}_{\Omega} \in \mathbb{R}^{3 \times 3}$ positive diagonal gain matrix, $k_{i \Omega}$ positive gain, $\eta_{3}$ a positive number, and $\boldsymbol{\Omega}_{r}$ defined by (10) combined with 34. If

$$
\frac{k}{k_{i \Omega}}\left\|\mathbf{Q}^{-\top}\right\| \bar{\epsilon}+\Delta_{2} \leq \Delta_{1}
$$

then, the following properties hold.

1) The error state $\left(\tilde{\mathbf{\Omega}}, \mathbf{I}_{\Omega}, \mathbf{R}^{\top} \mathbf{e}_{3}\right)$ converges either to $\left(\mathbf{0}, \mathbf{I}_{\Omega}^{\star}, \mathbf{e}_{3}\right)$ or $\left(\mathbf{0}, \mathbf{I}_{\Omega}^{\star},-\mathbf{e}_{3}\right)$ for all initial conditions, with $\mathbf{I}_{\Omega}^{\star} \triangleq k_{i \Omega}^{-1} \mathbf{Q}^{-\top} \overline{\boldsymbol{\Delta}}_{\Gamma}$.

2) The "desired" equilibrium $\left(\tilde{\mathbf{\Omega}}, \mathbf{I}_{\Omega}, \mathbf{R}^{\top} \mathbf{e}_{3}\right)=\left(\mathbf{0}, \mathbf{I}_{\Omega}^{\star}, \mathbf{e}_{3}\right)$ is almost-GAS and LES. The "undesired" equilibrium $\left(\tilde{\mathbf{\Omega}}, \mathbf{I}_{\Omega}, \mathbf{R}^{\top} \mathbf{e}_{3}\right)=\left(\mathbf{0}, \mathbf{I}_{\Omega}^{\star},-\mathbf{e}_{3}\right)$ is unstable.

The proof is given in Appendix A

\section{Comparative Simulation Results}

The proposed control approach applied to station keeping of fully-actuated AUVs without the need of linear velocity measurements is, in fact, inspired by the one proposed in [17, Remark 7] which corresponds to the particular case of using a downward-looking camera and which makes use of linear velocity measurements. Although the novel approach already has a practical advantage by depleting the need of a costly DVL, it is even more desirable if its performance is also comparable to the previous approach. Therefore, comparative simulation results of the two approaches using a realistic model of a fully-actuated AUV and in presence of a constant horizontal current $\mathbf{v}_{f}=\left[\frac{1}{2 \sqrt{2}}, \frac{1}{2 \sqrt{2}}, 0\right]^{\top}(m / s)$ will be reported thereafter. For convenience, let us call the proposed controller (c.f. Propositions 2, 3, 4) and the one proposed in [17] as Controller 1 and Controller 2, respectively.

\begin{tabular}{|c|c|c|c|}
\hline Specification & \multicolumn{3}{|c|}{ Numerical value } \\
\hline$m[k g]$ & \\
\hline$F_{b}[N]$ & \multirow{2}{*}{\multicolumn{3}{|c|}{$1.01 \mathrm{mg}$}} \\
\hline$l[\mathrm{~m}]$ & \multicolumn{2}{|c|}{0.025} & \\
\hline $\mathbf{r}_{C}[m]$ & \multicolumn{3}{|c|}{$\left[\begin{array}{lll}0 & 0 & 0.15\end{array}\right]$} \\
\hline \multirow{3}{*}{$\mathbf{J}_{0}\left[k g \cdot m^{2}\right]$} & 0.0842 & 0.004 & 0.005 \\
\hline & 0.004 & 0.2643 & 0.007 \\
\hline & 0.005 & 0.007 & 0.3116 \\
\hline \multirow{3}{*}{$\mathbf{M}_{A}^{22}\left[k g \cdot m^{2}\right]$} & 0.1 & 0.005 & 0.006 \\
\hline & 0.005 & 0.25 & 0.008 \\
\hline & 0.006 & 0.008 & 0.3 \\
\hline \multirow{3}{*}{$\mathbf{M}_{A}^{11}[k g]$} & 1.39 & 0.10 & 0.12 \\
\hline & 0.10 & 4.26 & 0.13 \\
\hline & 0.12 & 0.13 & 4.02 \\
\hline \multirow{3}{*}{$\mathbf{M}_{A}^{21}=\mathbf{M}_{A}^{12}$} & 0.002 & 0.02 & 0.01 \\
\hline & 0.02 & 0.003 & 0.018 \\
\hline & 0.01 & 0.018 & 0.003 \\
\hline $\mathbf{D}_{V l}\left[k g \cdot s^{-1}\right]$ & \multirow{4}{*}{\multicolumn{3}{|c|}{$\begin{array}{c}\operatorname{diag}(5.85,9.21,11.03) \\
\operatorname{diag}(36.57,57.58,68.97) \\
\operatorname{diag}(0.01126,0.01855,0.01701) \\
\operatorname{diag}(0.0053,0.0130,0.0118)\end{array}$}} \\
\hline $\mathbf{D}_{V q}\left[k g \cdot m^{-1}\right]$ & & & \\
\hline $\mathbf{D}_{\Omega l}\left[\mathrm{~kg} \cdot \mathrm{m}^{2} \cdot \mathrm{s}^{-1}\right]$ & & & \\
\hline $\mathbf{D}_{\Omega q}[N . m]$ & & & \\
\hline
\end{tabular}

The simulated vehicle is the BlueROV2 Its physical parameters are provided in Tab. [] where the added-mass, addedinertia and damping coefficients are roughly identified from

${ }^{2}$ http://bluerobotics.com/store/retired/bluerov-r1/
TABLE I: Specifications of the simulated AUV

\begin{tabular}{|c|c|}
\hline Controller & Gains and other parameters \\
\hline Outer-loop & $\mathbf{K}_{1}=\operatorname{diag}(3 s, 3 s, 3 s)$ \\
& $k_{2}=\frac{8}{3} \frac{s^{2}}{a^{\star}}, k_{3}=\frac{1}{3} \frac{s^{2}}{a^{\star}}, s=\sqrt{2}$ \\
& $k_{I}=0.7, \eta_{1}=1.8, \eta_{2}=2.3$ \\
& $k_{g}=1, k_{\Theta 1}=1, k_{\Theta 2}=\sqrt{2}, \Delta_{\Theta}=1$ \\
\hline Inner-loop & $\mathbf{K}_{\Omega}=\operatorname{diag}(3,3,3), k_{i \Omega}=2$ \\
& $k=10, \Delta_{1}=6.25, \Delta_{2}=2$ \\
& $a_{0}=0.5, k_{0}=20, \eta_{3}=8$ \\
\hline
\end{tabular}

TABLE II: Control gains and parameters of Controller 1

\begin{tabular}{|c|c|}
\hline Controller & Gains and other parameters \\
\hline Outer-loop & $k_{p 1}=0.4145, k_{p 2}=\sqrt{2}$ \\
& $k_{u}=1, k_{\Theta 1}=1, k_{\Theta 2}=\sqrt{2}, \Delta_{\Theta}=1$ \\
\hline Inner-loop & $\mathbf{K}_{V}=\operatorname{diag}(5,5,5), \mathbf{K}_{i V}=0.4 \mathbf{K}_{V}$ \\
& $\mathbf{K}_{\Omega}=\operatorname{diag}(3,3,3), k_{i \Omega}=2$ \\
& $k=10, \Delta_{1}=6.25, \Delta_{2}=2$ \\
& $a_{0}=0.5, k_{0}=20, \eta_{3}=8$ \\
\hline
\end{tabular}

TABLE III: Control gains and parameters of Controller 2

the given shape. To test the robustness of the controllers with respect to model uncertainties, the following "erroneous" estimates of $\mathbf{M}$ and $\mathbf{J}$ are used:

$$
\left\{\begin{aligned}
\hat{\mathbf{M}} & =m \mathbf{I}_{3}+\hat{\mathbf{M}}_{A}^{11}=\operatorname{diag}(8.712,12.712,10.816)[\mathrm{kg}] \\
\hat{\mathbf{J}} & =\hat{\mathbf{J}}_{0}+\hat{\mathbf{M}}_{A}^{22}=\operatorname{diag}(0.1642,0.5643,0.5116)\left[\mathrm{kg} \cdot \mathrm{m}^{2}\right]
\end{aligned}\right.
$$

One notes that these estimated parameters are quite different from the corresponding "real" ones.

The homography $\mathbf{H}$ is directly computed using (11) with $d^{\star}=1(m)$ and $\mathbf{n}^{\star}=\mathbf{R}_{\left\{\frac{\pi}{18}, \frac{\pi}{6}, 0\right\}} \mathbf{e}_{3}=$ $[0.4924,-0.1736,0.8529]^{\top}$. The initial conditions are $\mathbf{p}_{C}(0)=[-2,-1.5,-1]^{\top}(m), \mathbf{R}(0)=\mathbf{R}_{\left\{\frac{\pi}{18},-\frac{\pi}{18}, \pi\right\}}$, $\mathbf{V}(0)=\boldsymbol{\Omega}(0)=\mathbf{0}$. A very large initial yaw error is chosen (i.e. $\psi=\pi$ ) to verify the large stability domain of the control algorithms.

- Simulation with Controller 1 (i.e proposed approach): The proposed control approach including the outer-loop controller given in Propositions 2 -3 and the inner-loop controller given in Proposition 4 is simulated, with gains and parameters 


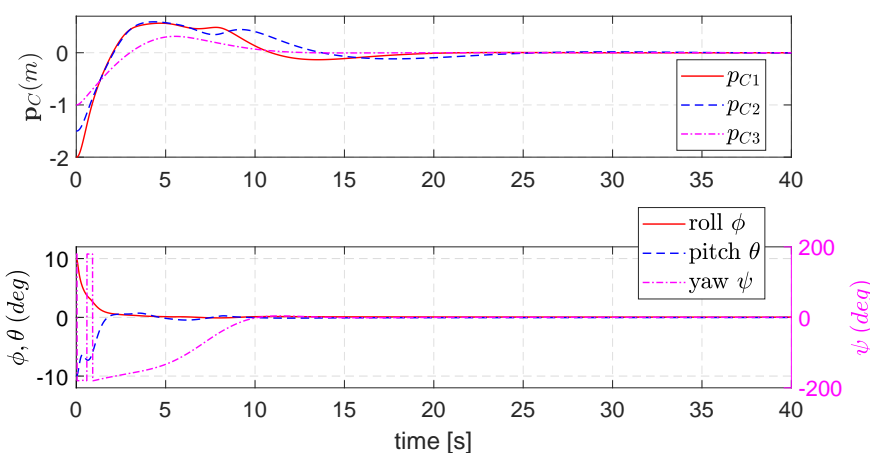

(a) Controller 1

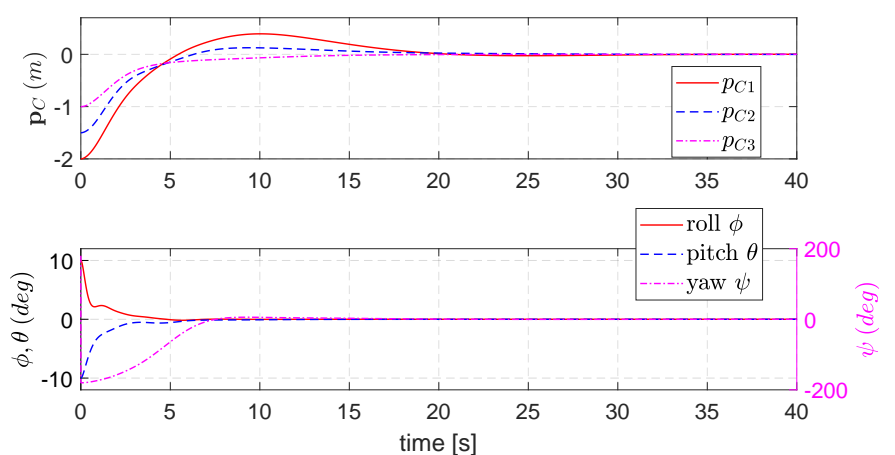

(b) Controller 2

Fig. 3: (Simulation) AUV's position and attitude (Euler angles) vs. time
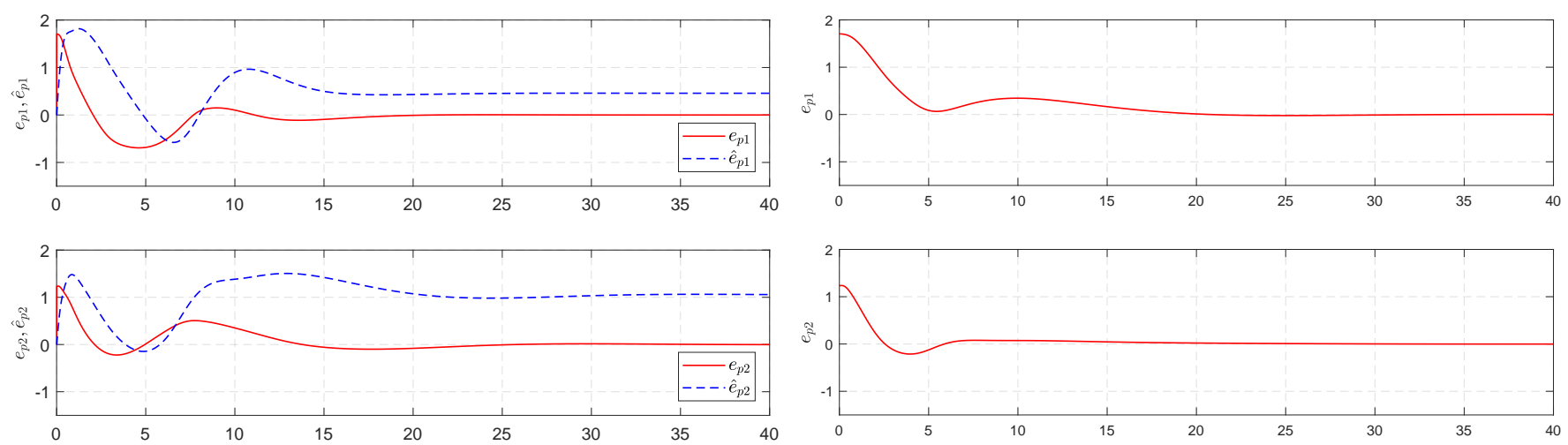

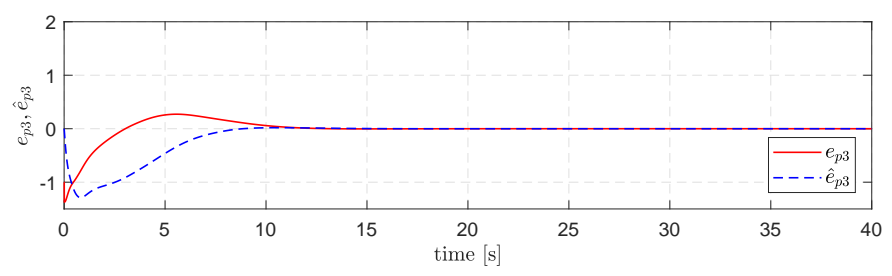

(a) Controller 1

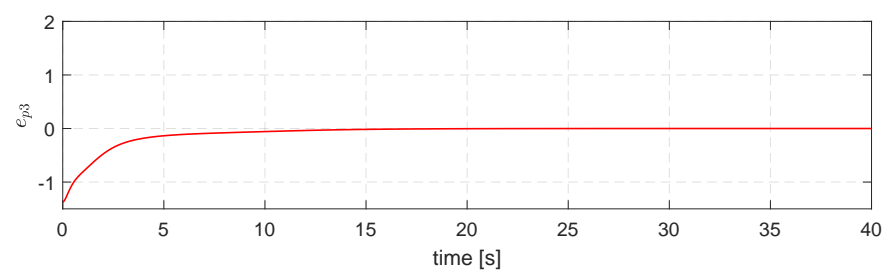

(b) Controller 2

Fig. 4: (Simulation) $\mathbf{e}_{p}$ (and $\hat{\mathbf{e}}_{p}$ ) vs. time

given in Tab. III The control gains have been obtained based on the classical pole placement technique using a coarse estimation of $a^{\star}$ equal to 1 . Due to the current velocity of significant magnitude (i.e. $0.5[\mathrm{~m} / \mathrm{s}]$ ), the drag force and Munk moment are not negligible. This shows the need of control robustification using, for instance, the integrator and high-gain observer techniques proposed in this paper. As observed from the simulation results reported in Figs. 3a 6a. the estimate $\hat{\boldsymbol{\Delta}}_{\Gamma}$ and the integrators in both inner- and outer-loop controllers successfully counteract for these abovementioned disturbances as well as the imprecise estimated system parameters. Fig. 3a shows the convergence of the AUV's position and orientation to zero without large overshoots. The sea current on horizontal plane has a clear impact on the integral variable $\mathbf{z}$, the estimate terms $\hat{\mathbf{e}}_{p}$ and $\hat{\boldsymbol{\Delta}}_{\Gamma}$, as well as the control force and torque. More precisely, $z_{1}, z_{2}, \hat{e}_{p 1}, \hat{e}_{p 2}$, $\hat{\Delta}_{\Gamma 3}, F_{c 1}, F_{c 2}$ and $\Gamma_{c 3}$ converge to non-null values as shown in Figs. $4 \mathrm{a}$ 6a. On the other hand, the vertical component of $F_{c 3}$ converges to a positive value since the vehicle is positively buoyant.

- Simulation with Controller 2 (i.e. approach proposed in
[17]): To make a fair comparison, the inner-loop controller of [17] has been revised by also incorporating a high-gain observer of the perturbation torque induced by sea current, similarly to the one proposed in Lemma 2 Control parameters and gains of the controller are given in Tab. IIII. They have been chosen so that the time evolution of the AUV's position has almost the same settling time as in the previous simulation. Simulation results are reported in Figs $3 \mathrm{~b} 6 \mathrm{~b}$. In overall, the time evolutions of the vehicle's pose and of the visual error $\mathbf{e}_{p}$ are quite reminiscent of the corresponding ones resulted by Controller 1. This implies that the performance of the proposed controller is comparable to our prior control approach [17] that in contrast relies on a costly DVL for linear velocity measurements.

\section{EXPERIMENTAL VALIDATIONS}

\section{A. Experimental setup}

The implementation of the proposed algorithm with realtime homography estimation has been performed on an inhouse AUV experimental platform (see Fig. 77. This platform has been developed based on a purchased BlueROV. 

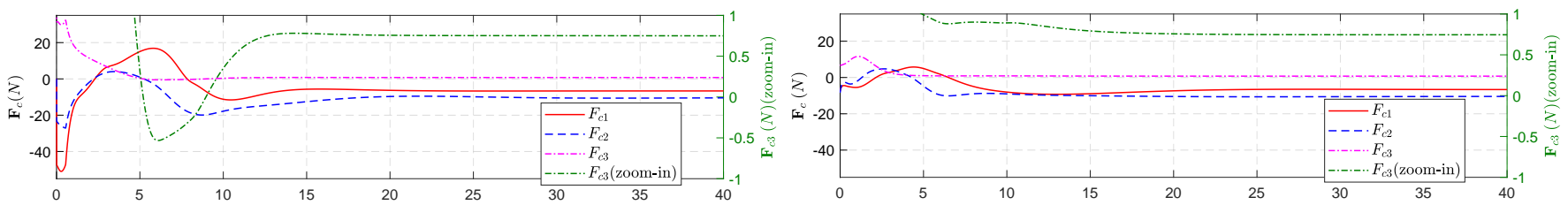

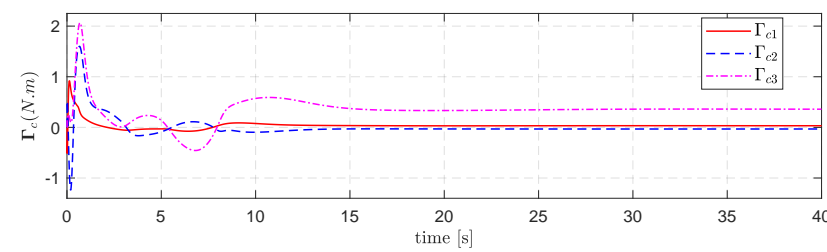

(a) Controller 1

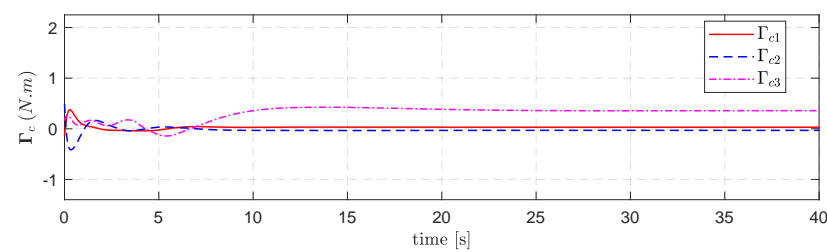

(b) Controller 2

Fig. 5: (Simulation) Control force $\mathbf{F}_{c}$ and torque $\boldsymbol{\Gamma}_{c}$ vs. time
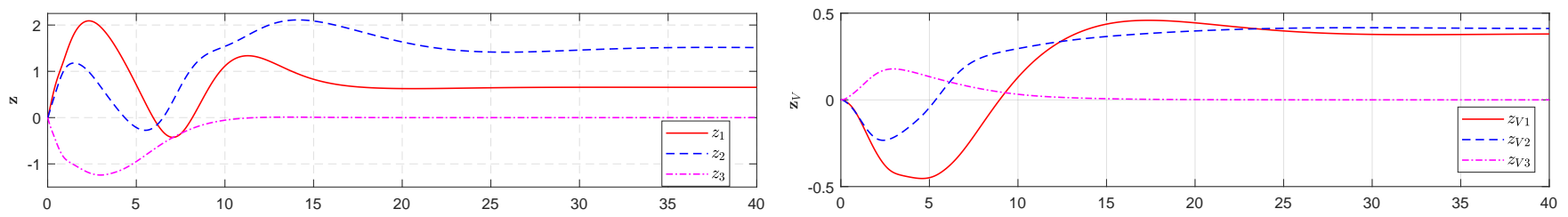

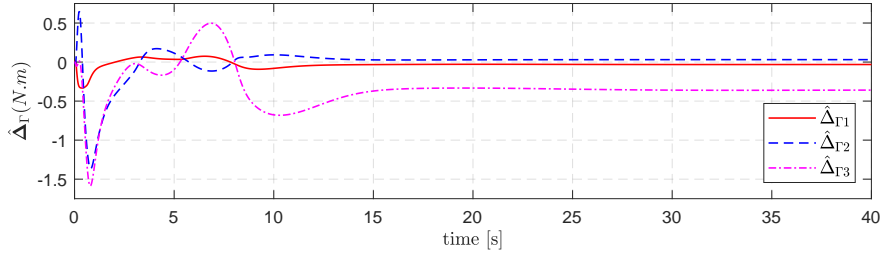

(a) Controller 1

Fig. 6: (Simulation) $\mathbf{z}$ (left), $\mathbf{z}_{V}$ (right), and $\hat{\Delta}_{\Gamma}$ vs. time
The on-board electronic components (see Fig. 8) include a widely-used 3DR Pixhawk flight controller and a compact and low-cost Odroid XU-4 octa-core single board computer. A laptop is used as a ground station, that is connected with Odroid XU-4 over the high speed Ethernet connection. In the AUV architecture depicted in Fig. 8, ROS plays the role of a middleware, providing communication (i.e. sending and receiving messages between the distributed components). The open-source PX4 autopilot widely-used for unmanned aerial vehicles is exploited and adapted for our research purpose.

The overall dimensions of the vehicle are $0.39[\mathrm{~m}] \times$ $0.33[\mathrm{~m}] \times 0.65[\mathrm{~m}]$ in height, width and length. The original dry weight of BlueROV is only $7.6[\mathrm{~kg}]$. However, for our research a longer tube has been used to accommodate the main electronic components and two smaller tubes have been added for camera and battery housing. After incorporating all these components, the total volume of the AUV is almost double to its original design. Consequently, the buoyancy force is significantly increased. To overcome that issue, additional weight blocks have been attached to the vehicle to achieve near neutral buoyancy. The weight blocks together with additional buoyant blocks give two degrees of freedom to adjust the center of mass $G$ and the center of buoyancy $B$ such that the line connecting them (i.e $B G$ ) is parallel with the direction of the downward-looking camera. The resulting total weight

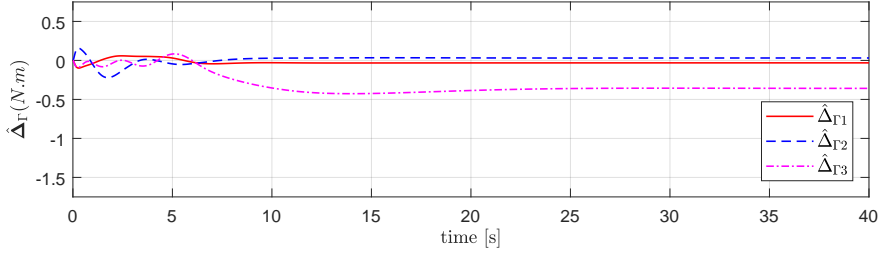

(b) Controller 2

of the AUV is approximately $16[\mathrm{~kg}]$ in air. The vehicle is equipped with three vertical thrusters for heave, pitch and roll actuations, two horizontal thrusters for yaw and surge actuations, and one lateral thruster for sway actuation.

For performing the real-time homography estimation, the AUV is equipped with a myAHRS+ IMU sensor providing measurement output at $100[\mathrm{~Hz}]$ and an oCam downward-looking monocular camera providing color images of $640[p x] \times 480[p x]$ at $20[H z]$. For homography estimation, we have used the HomographyLab library 3 wich has been developed by our team based on the homography observer proposed in [14]. This library has been implemented in $\mathrm{C}++$ combined with OpenCV for image processing (for instance, the FAST Feature Detector and ORB Descriptor Extractor algorithms available in the OpenCV library are employed for carrying out feature detection and descriptor extraction in images). HomographyLab has been evaluated at the TRL 7 (Technology Readiness Level) and is protected by the French Agency for the Protection of Programs. Real-time and robustness (with respect to fast camera motions, occlusions, image blurs, sudden changes in light intensity, poor image quality, etc.) are the two principle distinguished features of HomographyLab and the implemented algorithm with respect to the state-of-the-art codes and algorithms (see [14] for

3 http://sdb3.i3s.unice.fr/homographyLab/ 


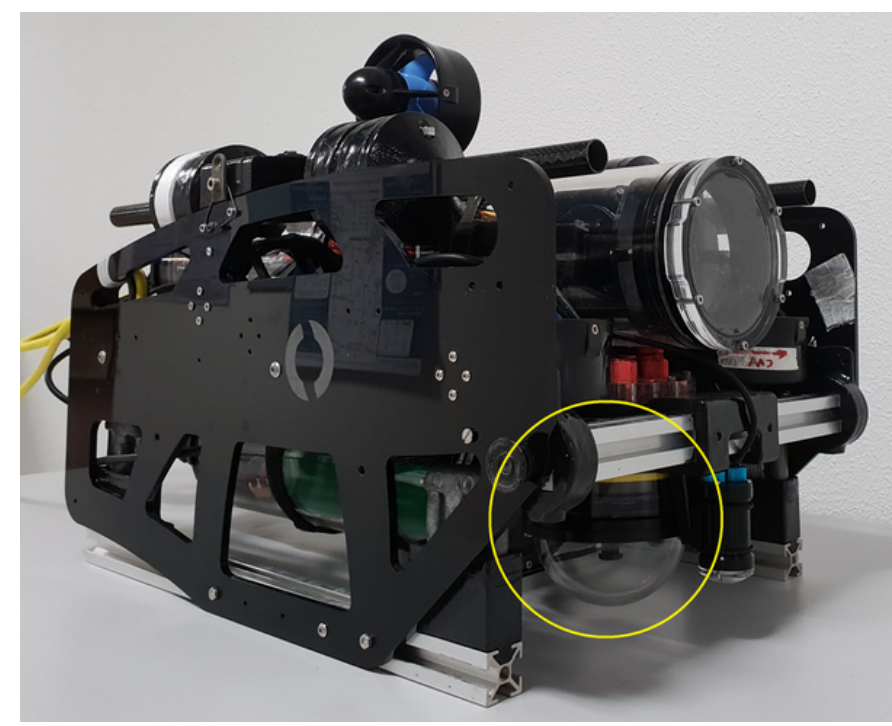

Fig. 7: AUV with downward-looking camera (inside yellow circle)

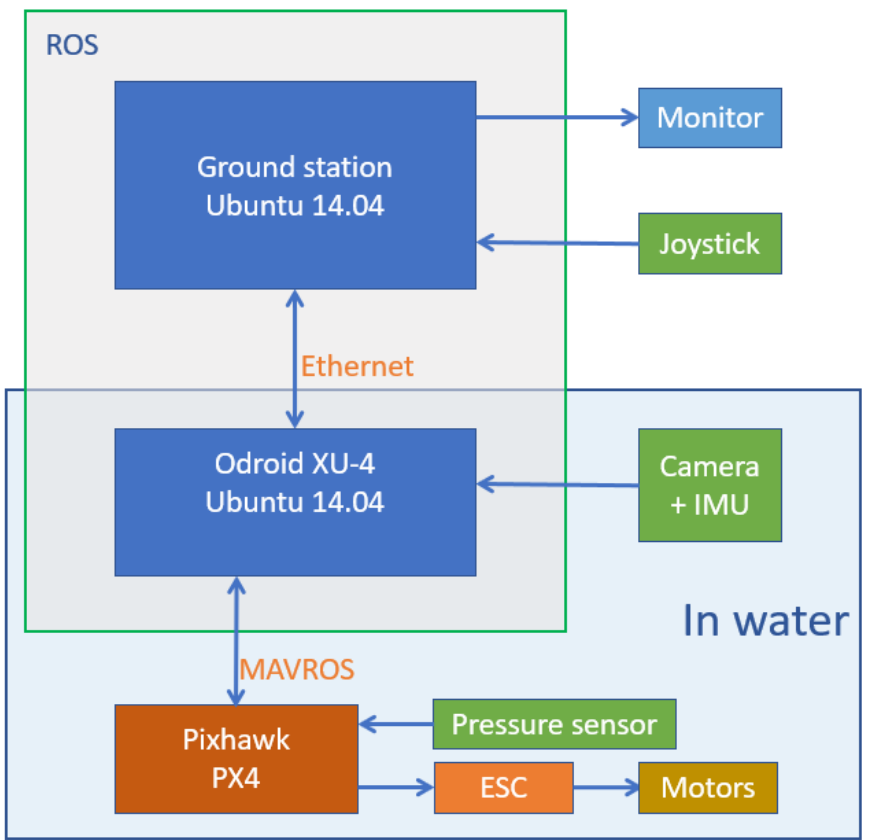

Fig. 8: AUV's architecture

more detail). In the reported experiments, HomographyLab has been implemented on the ground station laptop with an Intel Core i7-7700HQ octa-core CPU running at $2.8 \mathrm{GHz}$. The transmission of the data from the camera and the IMU to the ground station is carried out through the high speed Ethernet cable. The laptop has a Linux-based operating system and is responsible for the following tasks: 1) interfacing with the camera and IMU hardwares and acquisition of images and IMU data 2) real-time estimation of the homography at $10[\mathrm{~Hz}]$ 3 ) perform outer-loop control at $10[\mathrm{~Hz}]$, and 4) interfacing with the joystick and Pixhawk via Odroid to remotely control the vehicle.

To perform the station-keeping task, the UAV is initially in the teleoperation mode and is manually positioned at a

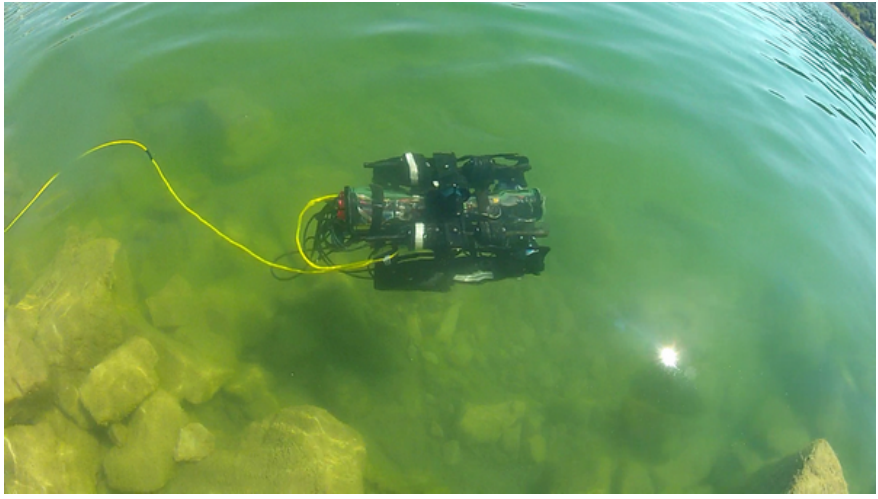

Fig. 9: Experimental validations in lake Saint-Cassien

certain depth above the lakebed so that the latter is visible by the camera ensuring a sufficient number of detected features for good homography estimation. Such unknown depth thus varies according to lighting conditions and water turbidity. In the reported experiment, the vehicle was positioned at about $0.5[\mathrm{~m}]$ above the lakebed.

The parameters and control gains involved in the computation of the control inputs are given in Tab. IV and Tab. V

\begin{tabular}{|c|c|}
\hline Specification & Numerical value \\
\hline$m[\mathrm{~kg}]$ & 16 \\
$F_{b}[\mathrm{~N}]$ & $1.01 \mathrm{mg}$ \\
$l[\mathrm{~m}]$ & 0.025 \\
$\mathbf{r}_{C}[\mathrm{~m}]$ & {$[0.200 .1]$} \\
$\hat{\mathbf{M}}=m \mathbf{I}_{3}+\hat{\mathbf{M}}_{A}^{11}[\mathrm{~kg}]$ & $\operatorname{diag}(17.868,23.868,21.024)$ \\
$\hat{\mathbf{J}}=\mathbf{J}_{0}+\hat{\mathbf{M}}_{A}^{22}\left[\mathrm{~kg} \cdot \mathrm{m}^{2}\right]$ & $\operatorname{diag}(0.3105,0.8486,1.0)$ \\
$\hat{\mathbf{D}}=m \mathbf{e}_{3 \times}[\mathrm{kg} \cdot \mathrm{m}]$ & $0.4 \mathbf{e}_{3 \times}$ \\
\hline
\end{tabular}

TABLE IV: Specifications of the experimental AUV

\section{B. Experimental results}

Experimental results carried out in lake Saint-Cassien (France) are reported next (see Fig. 97. Due to space limitation, only brief but most representative parts of total results are presented in the video https://youtu.be/p_oiISPOtgw. However, the reader can also view two other videos showing the capability of long-term station keeping (more than 30 minutes) as well as other trials carried out during our research process with different water turbidity conditions and target images:

- https://youtu.be/mkAAjX3mgVk

- https://youtu.be/KjAfYu1jG18

Regarding the reported video, experimental results including the time evolution of the control force $\mathbf{F}_{c}$, the visual error $\mathbf{e}_{p}$ and its estimate $\hat{\mathbf{e}}_{p}$, the homography component $h_{12}$, and

\begin{tabular}{|c|c|}
\hline Controller & Gains and other parameters \\
\hline Proposition 2 & $\mathbf{K}_{1}=\operatorname{diag}(3 s, 3 s, 3 s)$ \\
& $k_{2}=\frac{8}{3} \frac{s^{2}}{a^{\star}}, k_{3}=\frac{1}{3} \frac{s^{2}}{a^{\star}}, \quad s=0.9$ \\
& $k_{I}=0.1, \eta_{1}=1.8, \eta_{2}=2.3$ \\
\hline Proposition 3 & $k_{g}=1, k_{\Theta 1}=1 / 2, k_{\Theta 2}=\sqrt{2}, \Delta_{\Theta}=2 \sqrt{2}$ \\
\hline Proposition 4 & $\begin{array}{c}\mathbf{K}_{\boldsymbol{\Omega}}=\operatorname{diag}(1.863,5.0916,5.0), \mathbf{K}_{i \Omega}=0.1 \mathbf{K}_{\boldsymbol{\Omega}} \\
a_{0}=0.5, k_{0}=20, \eta_{3}=6\end{array}$ \\
\hline
\end{tabular}

TABLE V: Control gains and parameters in experiment 

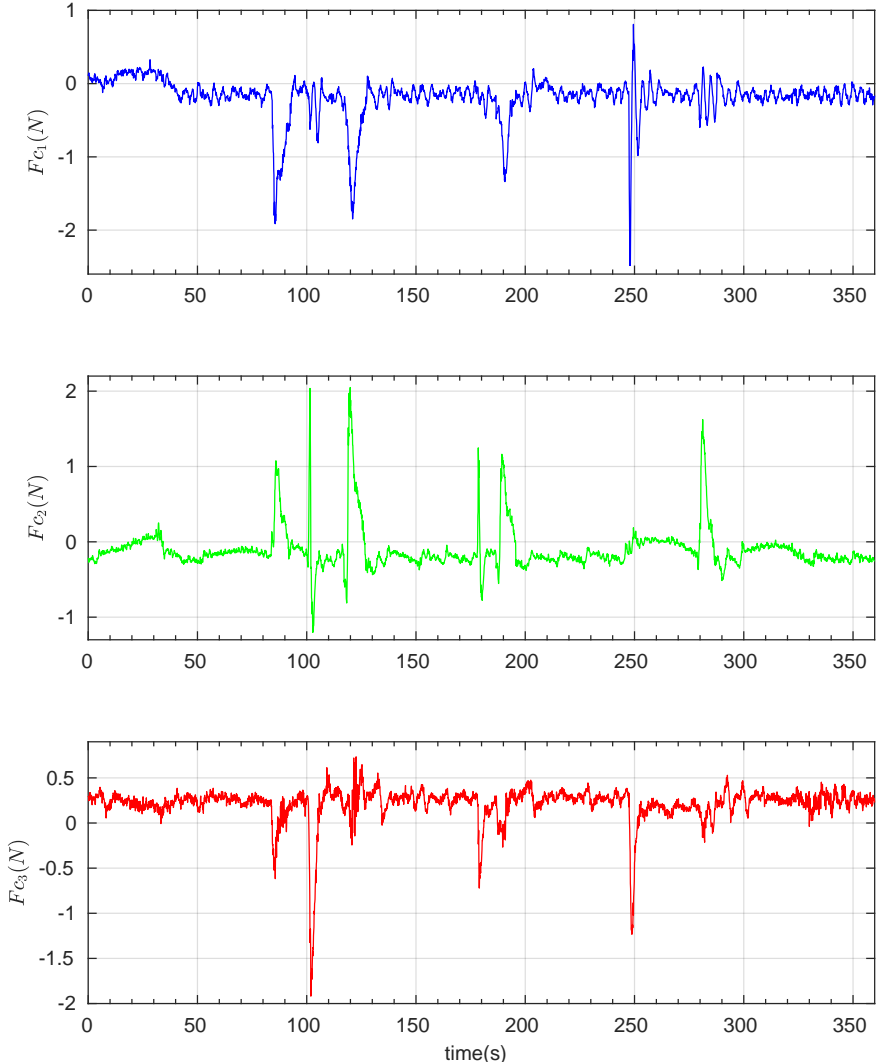

Fig. 10: Control force $\mathbf{F}_{c}$ vs. time

the Frobenius norm of $\mathbf{H}-\mathbf{I}$ are shown in Figs. 10,13 respectively.

To excite the external force response, the AUV has been manually moved by a stick at time instants $85[s], 100[s]$, $120[s], 180[s], 245[s]$ and $280[s]$. In Fig. 11 one observes that right after finishing the interaction, the AUV went back to its stabilized pose with relatively small overshoot. The control force generated for pushing the AUV back to the reference position is shown in Fig. 10. The settling time is about 30 seconds. The transient response also shows the efficiency of the integrator in eliminating the static error caused by the current.

In general, one can clearly observe the practical convergence of the AUV's pose to the desired one as illustrated by the practical convergence of the Frobenius norm $\|\mathbf{H}-\mathbf{I}\|$ to zero (see Fig. 13). In particular, the convergence of the AUV's position is attested by the convergence of visual error $\mathbf{e}_{p}$ to zero (Fig. 11) whereas the component $h_{12}$ converges near to zero (Fig. 12) showing the practical convergence of the AUV's yaw angle to the desired one.

The effectiveness of the integrator correction in dealing with unknown currents and model uncertainties can be appreciated from Figs. 10 and 11. In steady state, the horizontal components of the control force $F_{c 1}$ and $F_{c 2}$ (Fig. 10) converge to non-zero values required to compensate for the horizontal current. The vertical component $F_{c 3}$ ultimately remains far from zero since the AUV is positively buoyant. In Fig. 11 it can be seen that $\mathbf{e}_{p}$ converges near to zero and that the offsets between the corresponding components of $\mathbf{e}_{p}$ and $\hat{\mathbf{e}}_{p}$
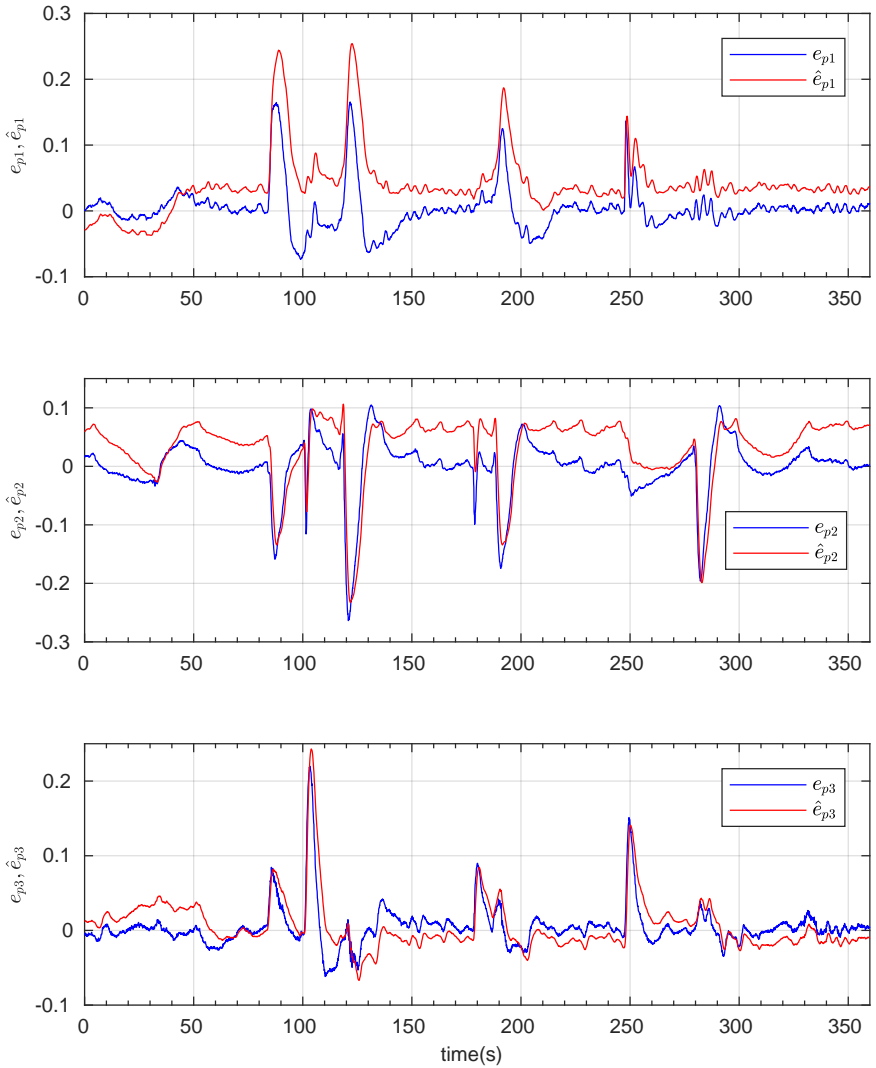

Fig. 11: $\mathbf{e}_{p}$ and $\hat{\mathbf{e}}_{p}$ vs. time

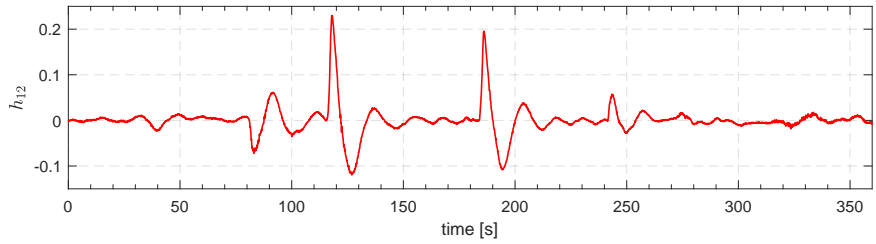

Fig. 12: $h_{12}$ vs. time

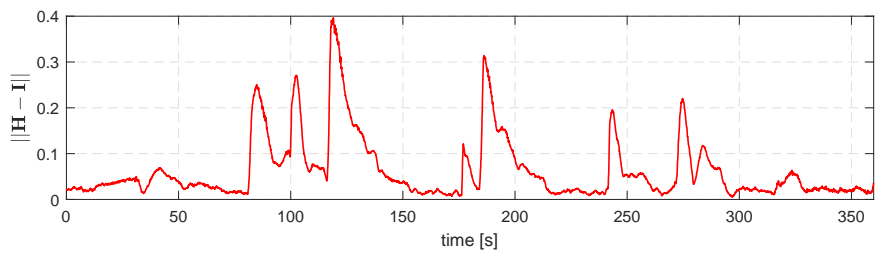

Fig. 13: Frobenius norm $\|\mathbf{H}-\mathbf{I}\|$ vs. time. The AUV has been pushed by a stick at time instants $85[s], 100[s], 120[s], 180[s]$, $245[s]$ and $280[s]$. One observes the practical convergence of $\|\mathbf{H}-\mathbf{I}\|$ to zero after these instants.

are almost constant in steady state.

As can be observed from the reported video, the experiment has been carried out on a sunny day and the waves generate moving bright spots in the lakebed that periodically alter local brightness of the images captured. However, very good and robust quality of homography estimation can be appreciated despite that change in light illumination as well as large occlusions due to a panel fixed to the stick in between $310[s]$ and $360[s]$. It is also worth noting that, theoretically, the homography estimation uses a planar target. In the experiment, this assumption does not hold true since the lakebed is covered 
by $3 \mathrm{D}$ reliefs such as sand rocks, and additionally the camera is relatively close to the lakebed. However, the proposed controller along with real-time homography estimation keeps working efficiently, showing the robustness of the control approach.

One can observe some small oscillations during the convergence. This is essentially due to the fact that the outer-loop control runs at a low frequency $(10[H z])$ and also due to the imperfect thrust generated by the thrusters inside its deadband. This phenomenon becomes more prominent when the outer-loop poles are large. Hence, smaller pole values have been chosen while tuning the gains in the actual experiment as compared to the ones used in the reported simulation.

In conclusion, the experimental results for the overall control approach (i.e inner and outer-loop controls) are quite convincing even though some vehicle's physical parameters (e.g. added mass and added moment of inertia) are only roughly estimated and the AUV was subjected to various challenging conditions (e.g. significant unknown currents, water turbidity, lightning variation, target occlusion, etc.).

\section{CONCLUSION}

In this paper a homography-based dynamic control approach of fully-actuated underwater vehicles is proposed. An advanced feature with respect to existing works on the topic is the non-requirement of a costly DVL for linear velocity measurements. A potential application to station keeping has been demonstrated in a real environment with very encouraging results despite challenging conditions such as important disturbances induced by currents, water turbidity, lightning variation, target occlusion, etc. The proposed approach will undoubtedly enlarge the working envelop of low-cost remotely operated underwater vehicles (ROVs) as a costly DVL is not required. Even for industrial or professional ROVs and AUVs equipped with DVLs, the approach also allows for overcoming the common problem of measurement imprecision of DVLs due to threshold violation when operating close to underwater structures or a sea bottom. A potential extension of this work concerns the station-keeping task of an AUV equipped with a forward-looking camera observing a (near) vertical target.

Acknowledgement: This work was supported by the French ANR Astrid CONGRE (ANR-18-ASTR-0006) and FUI GreenExplorer projects.

\section{APPENDIX}

\section{ProOF OF PROPOSITION 4}

Proof: Consider the positive storage function

$$
\mathcal{V} \triangleq \begin{cases}\frac{1}{2}|\mathbf{Q} \tilde{\boldsymbol{\Omega}}|^{2} & \text { if }|\mathbf{Q} \tilde{\boldsymbol{\Omega}}| \leq \Delta_{2} \\ \frac{1}{2}\left(2|\mathbf{Q} \tilde{\boldsymbol{\Omega}}|-\Delta_{2}\right) \Delta_{2} & \text { otherwise }\end{cases}
$$

Using the fact that $\mathbf{J}=\mathbf{Q}^{\top} \mathbf{Q}$ one verifies that

$$
\dot{\mathcal{V}}=\min \left(1, \frac{\Delta_{2}}{|\mathbf{Q} \tilde{\mathbf{\Omega}}|}\right) \tilde{\boldsymbol{\Omega}}^{\top} \mathbf{J} \dot{\tilde{\mathbf{\Omega}}}
$$

From (36), (38) and the definition of $\tilde{\boldsymbol{\Omega}}$ one obtains

$$
\begin{aligned}
\mathbf{J} \dot{\tilde{\mathbf{\Omega}}}= & (\mathbf{J} \boldsymbol{\Omega})_{\times} \tilde{\mathbf{\Omega}}-\operatorname{sat}^{\eta_{3}}\left(\mathbf{K}_{\Omega} \tilde{\boldsymbol{\Omega}}\right)+\max \left(1, \frac{|\mathbf{Q} \tilde{\boldsymbol{\Omega}}|}{\Delta_{2}}\right) \boldsymbol{\Gamma}_{g} \\
& -k_{i \Omega} \mathbf{Q}^{\top} \mathbf{I}_{\Omega}+\overline{\boldsymbol{\Delta}}_{\Gamma}
\end{aligned}
$$

Consider the Lyapunov function candidate

$$
\mathcal{L}_{1} \triangleq \mathcal{V}+m g l\left(1-\mathbf{e}_{3}^{\top} \mathbf{R}^{\top} \mathbf{e}_{3}\right)+\frac{1}{2} \mathbf{k}_{i \Omega}\left|\tilde{\mathbf{I}}_{\Omega}\right|^{2}
$$

with $\tilde{\mathbf{I}}_{\Omega} \triangleq \mathbf{I}_{\Omega}-\mathbf{I}_{\Omega}^{\star}$. Calculating the time-derivative of $\mathcal{L}_{1}$ using the expression 40 of $\dot{\mathcal{V}}$, equation 36 , the torque control expression (38), the definition (10) of $\boldsymbol{\Omega}_{r}$, the expression (37) of $\dot{\mathbf{I}}_{\Omega}$ and the fact that $\operatorname{sat}^{\Delta_{2}}(\mathbf{Q} \tilde{\boldsymbol{\Omega}})=\min \left(1, \frac{\Delta_{2}}{|\mathbf{Q} \tilde{\boldsymbol{\Omega}}|}\right) \mathbf{Q} \tilde{\boldsymbol{\Omega}}$, one deduces

$$
\begin{aligned}
\dot{\mathcal{L}}_{1}= & -\min \left(1, \frac{\Delta_{2}}{|\mathbf{Q} \tilde{\boldsymbol{\Omega}}|}\right) \tilde{\boldsymbol{\Omega}}^{\top} \operatorname{sat}^{\eta_{3}}\left(\mathbf{K}_{\Omega} \tilde{\boldsymbol{\Omega}}\right)-\mathbf{k}_{i \Omega} \tilde{\mathbf{I}}_{\Omega}^{\top} \operatorname{sat}^{\Delta_{2}}(\mathbf{Q} \tilde{\boldsymbol{\Omega}}) \\
& -m g l \boldsymbol{\Omega}_{r}^{\top} \mathbf{e}_{3} \times \mathbf{R}^{\top} \mathbf{e}_{3}+\mathbf{k}_{i \Omega} \tilde{\mathbf{I}}_{\Omega}^{\top} \dot{\mathbf{I}}_{\Omega} \\
= & -\min \left(1, \frac{\Delta_{2}}{|\mathbf{Q} \tilde{\boldsymbol{\Omega}}|}\right) \tilde{\boldsymbol{\Omega}}^{\top} \operatorname{sat}^{\eta_{3}}\left(\mathbf{K}_{\Omega} \tilde{\boldsymbol{\Omega}}\right)-m g l k_{g}\left|\mathbf{e}_{3} \times \mathbf{R}^{\top} \mathbf{e}_{3}\right|^{2} \\
& -k k_{i \Omega}\left|\tilde{\mathbf{I}}_{\Omega}\right|^{2}+k_{i \Omega} \tilde{\mathbf{I}}_{\Omega}^{\top}\left(-k \mathbf{I}_{i \Omega}^{\star}-\operatorname{sat}^{\Delta_{2}}(\mathbf{Q} \tilde{\boldsymbol{\Omega}})\right. \\
& \left.+\operatorname{sat}^{\Delta_{1}}\left(k \tilde{\mathbf{I}}_{\Omega}+k \mathbf{I}_{\Omega}^{\star}+\operatorname{sat}^{\Delta_{2}}(\mathbf{Q} \tilde{\boldsymbol{\Omega}})\right)\right) \\
\leq & -\min \left(1, \frac{\Delta_{2}}{|\mathbf{Q} \tilde{\boldsymbol{\Omega}}|}\right) \tilde{\boldsymbol{\Omega}}^{\top} \operatorname{sat}^{\eta_{3}}\left(\mathbf{K}_{\Omega} \tilde{\boldsymbol{\Omega}}\right)-m g l k_{g}\left|\mathbf{e}_{3} \times \mathbf{R}^{\top} \mathbf{e}_{3}\right|^{2}
\end{aligned}
$$

where the last inequality is obtained using condition $(39)$ and the fact that $\forall a, b \in \mathbb{R}^{3}, \Delta \in \mathbb{R}^{+}$one has $\mid-a+\operatorname{sat}^{\Delta}(b+$ $a)|\leq| b \mid$ if $|a| \leq \Delta$ (see [12] for the proof). Clearly $\dot{\mathcal{L}}_{1}$ is negative semi-definite. Remark that system (41) and (37) is not autonomous due to the time-varying term $\Omega$ and consequently La Salle's principle does not apply. However, $\tilde{\boldsymbol{\Omega}}$ and $\mathbf{I}_{\Omega}$ are bounded with respect to initial conditions. Since $\boldsymbol{\Omega}_{r}$ and its derivative are bounded thanks to the expressions (10) and (34), one deduces from 41 that $\dot{\tilde{\Omega}}$ is also bounded. Then it is straightforward to verify that $\ddot{\mathcal{L}}$ is also bounded, implying the uniform continuity of $\dot{\mathcal{L}}$. Then, application of Barbalat's lemma ensures that $\dot{\mathcal{L}}$ and, thus, $\tilde{\boldsymbol{\Omega}}$ and $\mathbf{e}_{3} \times \mathbf{R}^{\top} \mathbf{e}_{3}$ converge to zero. Next, using Barbalat-like arguments it can be shown that $\dot{\tilde{\Omega}}$ also converges to zero, implying the convergence of $\mathbf{I}_{\Omega}$ to $\mathbf{I}_{\Omega}^{\star}$. The convergence of $\mathbf{e}_{3} \times \mathbf{R}^{\top} \mathbf{e}_{3}$ to zero implies that $\mathbf{R e}_{3}$ converges to either $\mathbf{e}_{3}$ or $-\mathbf{e}_{3}$. So far we have proved that $\left(\tilde{\boldsymbol{\Omega}}, \mathbf{I}_{\Omega}, \mathbf{R e}_{3}\right)$ converges either to $\left(\mathbf{0}, \mathbf{I}_{\Omega}^{\star}, \mathbf{e}_{3}\right)$ or $\left(\mathbf{0}, \mathbf{I}_{\Omega}^{\star},-\mathbf{e}_{3}\right)$.

It remains to show that the "desired" equilibrium $\left(\tilde{\boldsymbol{\Omega}}, \mathbf{I}_{\Omega}, \mathbf{R e}_{3}\right)=\left(\mathbf{0}, \mathbf{I}_{\Omega}^{\star}, \mathbf{e}_{3}\right)$ is LES and the "undesired" equi$\operatorname{librium}\left(\tilde{\boldsymbol{\Omega}}, \mathbf{I}_{\Omega}, \mathbf{R e}_{3}\right)=\left(\mathbf{0}, \mathbf{I}_{\Omega}^{\star},-\mathbf{e}_{3}\right)$ is unstable. Note that the almost-GAS of the "desired" equilibrium then directly follows. In the first-order approximations, one has $\mathbf{R} \approx \mathbf{I}+\boldsymbol{\Theta}_{\times}$with $\boldsymbol{\Theta}=[\phi, \theta, \psi]^{\top}$ and, subsequently, $\mathbf{e}_{3 \times} \mathbf{R}^{\top} \mathbf{e}_{3} \approx[-\phi,-\theta, 0]^{\top}$. Denoting $\left[\tilde{\omega}_{1}, \tilde{\omega}_{2}, \tilde{\omega}_{3}\right]^{\top} \triangleq \tilde{\boldsymbol{\Omega}}$ and using the approximation $\dot{\Theta} \approx \Omega$, one obtains the following linearized system of 36 and 37

$$
\left\{\begin{array}{l}
\dot{\phi} \approx \tilde{\omega}_{1}-k_{g} \phi \\
\dot{\theta} \approx \tilde{\omega}_{2}-k_{g} \theta \\
\dot{\tilde{\mathbf{\Omega}}} \approx-\mathbf{J}^{-1} \mathbf{K}_{\Omega} \tilde{\boldsymbol{\Omega}}-\mathbf{J}^{-1} k_{i \Omega} \mathbf{Q}^{\top} \tilde{\mathbf{I}}_{\Omega}+m g l \mathbf{J}^{-1}[-\phi,-\theta, 0]^{\top} \\
\dot{\mathbf{I}}_{\Omega}=\mathbf{Q} \tilde{\boldsymbol{\Omega}}
\end{array}\right.
$$

Consider the following Lyapunov function candidate 


$$
\mathcal{L}_{\Omega}=\frac{1}{2} \tilde{\boldsymbol{\Omega}}^{\top} \mathbf{J} \tilde{\boldsymbol{\Omega}}+\frac{1}{2} \mathbf{k}_{i \Omega}\left|\tilde{\mathbf{I}}_{\Omega}\right|^{2}+\frac{1}{2} m g l\left(\phi^{2}+\theta^{2}\right)
$$

One verifies that

$$
\dot{\mathcal{L}}_{\Omega}=-\tilde{\boldsymbol{\Omega}}^{\top} \mathbf{K}_{\Omega} \tilde{\boldsymbol{\Omega}}-k_{g} m g l\left(\phi^{2}+\theta^{2}\right) \leq 0
$$

From here, LaSalle's principle ensures that $\tilde{\boldsymbol{\Omega}}, \phi$ and $\theta$ and, thus, $\dot{\tilde{\Omega}}$ converge to zero, which implies the convergence of $\mathbf{z}_{\Omega}$ to zero. The convergence of $\phi$ and $\theta$ to zero is equivalent to the convergence of $\mathbf{R}^{\top} \mathbf{e}_{3}$ to $\mathbf{e}_{3}$. Since the equilibrium $\left(\tilde{\boldsymbol{\Omega}}, \mathbf{I}_{\Omega}, \mathbf{R}^{\top} \mathbf{e}_{3}\right)=\left(\mathbf{0}, \mathbf{0}, \mathbf{e}_{3}\right)$ of the linearized system (44) is asymptotically stable, it is also exponentially stable.

Now, the Chetaev's theorem is used to prove the instability of the equilibrium $\left(\tilde{\boldsymbol{\Omega}}, \mathbf{I}_{\Omega}, \mathbf{R}^{\top} \mathbf{e}_{3}\right)=\left(\mathbf{0}, \mathbf{0},-\mathbf{e}_{3}\right)$. Define $\mathbf{y}=$ $\mathbf{e}_{3}+\mathbf{R}^{\top} \mathbf{e}_{3}$. Consider the positive function $\mathcal{S}_{1}(\mathbf{y}) \triangleq \mathbf{y}^{\top} \mathbf{e}_{3}=$ $1+\mathbf{e}_{3}^{\top} \mathbf{R}^{\top} \mathbf{e}_{3}$, satisfying $\mathcal{S}_{1}(\mathbf{0})=0$. Define $U_{r} \triangleq\left\{\mathbf{y} \mid \mathcal{S}_{1}(\mathbf{y})>\right.$ $0,|\mathbf{y}|<r\}$ for some number $0<r<1$. Note that $U_{r}$ is nonempty. By neglecting all high-order terms, one verifies that

$$
\dot{\mathcal{S}}_{1} \approx \mathbf{e}_{3}^{\top} \mathbf{R} \boldsymbol{\Omega}_{r \times} \mathbf{e}_{3}=k_{g}\left|\mathbf{e}_{3 \times} \mathbf{R}^{\top} \mathbf{e}_{3}\right|^{2}=k_{g}\left|\mathbf{e}_{3 \times} \mathbf{y}\right|^{2}
$$

For all $\mathbf{y} \in U_{r}$, the fact that $\mathbf{y}^{\top} \mathbf{e}_{3}>0$ is equivalent to $\left|\mathbf{e}_{3 \times} \mathbf{y}\right|^{2}>0$, which implies that $\dot{\mathcal{S}}_{1}>0$. Since all conditions of Chetaev's theorem are now united [16], one concludes that the origin of the linearized system about the undesired equilibrium (so that $\mathbf{y}=\mathbf{0}$ ) is unstable.

\section{REFERENCES}

[1] G. Allibert, M.-D. Hua, S. Krupínski, and T. Hamel. Pipeline following by visual servoing for Autonomous Underwater Vehicles. Control Engineering Practice, 82:151-160, 2019.

[2] J. Antich and A. Ortiz. Development of the control architecture of an underwater cable tracker. Int. J. of Intelligent Systems, 20(5):477-498, 2005.

[3] S. Benhimane and E. Malis. Homography-based 2D visual tracking and servoing. Int. J. of Robotics Research, 26(7):661-676, 2007.

[4] L. Brignone, M. Perrier, and C. Viala. A fully autonomous docking strategy for intervention AUVs. In MTS/IEEE OCEANS'07, pages 1-6, 2007.

[5] F. Chaumette and S. Hutchinson. Visual servo control, Part II: Advanced approaches. IEEE Robotics and Automation Mag., 14(1):109-118, 2007.

[6] X. Cufi, R. Garcia, and P. Ridao. An approach to vision-based station keeping for an unmanned underwater vehicle. In IEEE/RSJ Int. Conf. on Intelligent Robots \& Systems, 2002.

[7] H. de Plinval, P. Morin, and P. Mouyon. Stabilization of a class of underactuated vehicles with uncertain position measurements and application to visual servoing. Automatica, Elsevier, 77(0):155-169, 2017.

[8] R. Garcia, J. Batlle, X. Cufi, and J. Amat. Positioning an underwater vehicle through image mosaicking. In IEEE Int. Conf. on Robotics \& Automation, 2001.

[9] T. F. Gonçalves, J. R. Azinheira, and P. Rives. Vision-based autonomous approach and landing for an aircraft using a direct visual tracking method. In Int. Conf. on Informatics in Control, Automation and Robotics, pages 94-101, 2009.

[10] T. Hamel and R. Mahony. Visual servoing of an under-actuated dynamic rigid-body system: an image-based approach. IEEE Trans. on Robotics and Automation, 18(2):187-198, 2002.

[11] R. Hartley and A. Zisserman. Multiple View Geometry in Computer Vision. Cambridge Univ. Press, 2003.

[12] M.-D. Hua. Contributions to the automatic control of aerial vehicles. $\mathrm{PhD}$ thesis, 2009.

[13] M.-D. Hua, T. Hamel, P. Morin, and C. Samson. Balanced-force-control of underactuated thrust-propelled vehicles. 46th IEEE Conference on Decision and Control (CDC), pages 6435-6441, 2007.

[14] M.-D. Hua, J. Trumpf, T. Hamel, R. Mahony, and P. Morin. Featurebased recursive observer design for homography estimation and its application to image stabilization. Asian Journal of Control, pages 1-16, 2019.

[15] S. Hutchinson, G. Hager, and P. Cork. A tutorial on visual servo control IEEE Transactions on Robotics and Automation, 12(5):651-670, 1996.
[16] H. K. Khalil. Nonlinear systems (3rd Edition). Prentice Hall, 2002.

[17] S. Krupínski, G. Allibert, M.-D. Hua, and T. Hamel. An inertialaided homography-based visual servoing control approach for (almost) fully actuated autonomous underwater vehicles. IEEE Transactions on Robotics, 33(5):1041-1060, 2017.

[18] N. E. Leonard. Stability of a bottom-heavy underwater vehicle. Automatica, 33(3):331-246, 1997.

[19] J.-E Lots, D. M. Lane, E. Trucco, and F. Chaumette. A 2-D visual servoing for underwater vehicle station keeping. In IEEE Int. Conf. on Robotics and Automation (ICRA), pages 2767-2772, 2001.

[20] K. N. Lwin, N. Mukada, M. Myint, D. Yamada, A. Yanou, T. Matsuno, K. Saitou, W. Godou, T. Sakamoto, and M. Minami. Visual docking against bubble noise with 3-D perception using dual-eye cameras. IEEE Journal of Oceanic Engineering, Early Access:1-24, 2018.

[21] Y. Ma, J. Kosecka, and S. Sastry. Vision guided navigation for a nonholonomic mobile robot. IEEE Transactions on Robotics and Automation, 15(3):521-536, 1999.

[22] F. Maire, D. Prasser, M. Dunbabin, and M. Dawnson. A vision based target detection system for docking of an autonomous underwater vehicle. In Australasian Conference on Robotics and Automation (ACRA), 2009.

[23] E. Malis, F. Chaumette, and S. Boudet. 2 1/2D visual servoing. IEEE Trans. on Robotics and Automation, 15(2):238-250, 1999.

[24] E. Malis and M. Vargas. Deeper understanding of the homography decomposition for vision-based control. INRIA Researh report, RR6303:90, 2007.

[25] S. Matsumoto and Y. Ito. Real-time vision-based tracking of submarinecables for AUV/ROV. In Challenges of Our Changing Global Environment. MTS/IEEE OCEANS'95, pages 1997-2002, 1995.

[26] A. Micaelli and C. Samson. Trajectory tracking for unicycle-type and two-steering-wheels mobile robots. Technical Report 2097, INRIA, 1993.

[27] L.-H. Nguyen, M.-D. Hua, G. Allibert, and T. Hamel. Inertialaided homography-based visual servo control of autonomous underwater vehicles without linear velocity measurements. In 21st International Conference on System Theory, Control and Computing (ICSTCC), pages 9-16, 2017.

[28] N. Palomeras, S. Nagappa, D. Ribas, N. Gracias, and M. Carreras. Vision-based localization and mapping system for AUV intervention. In MTS/IEEE OCEANS'13, pages 1-7, 2013.

[29] E. Panteley and A. Loria. On global uniform asymptotic stability of non linear time-varying non autonomous systems in cascade. System \& Control Letters, 33(2):131-138, 1998.

[30] P. Rives and J.-J. Borrelly. Underwater pipe inspection task using visual servoing techniques. In IEEE Int. Conf. on Intelligent Robots and Systems (IROS), pages 63-68, 1997.

[31] S. van der Zwaan, A. Bernardino, and J. Santos-Victor. Visual station keeping for floating robots in unstructured environments. Robotics and Autonomous Systems, 39(3):145-155, 2002.

[32] M. Wirtz, M. Hildebrandt, and C. Gaudig. Design and test of a robust docking system for hovering AUVs. In MTS/IEEE OCEANS'12, pages $1-6,2012$.

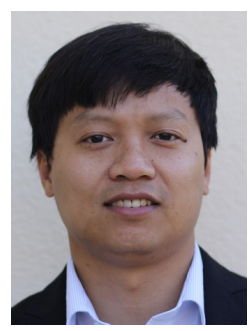

Lam-Hung Nguyen obtained his Engineer degree from Bauman Moscow State Technical University, Russia in 2007, then received his MSc degree from Institut Supérieur de l'Aéronautique et l'Espace (ISAE-SUPAERO), France in 2014, and the PhD degree from the University Côte d'Azur, France in 2019. Currently, he is a post-doctoral researcher at the I3S UCA-CNRS Laboratory, working on transferring the HomographyLab software to ECA Robotics. His research interests include nonlinear control theory and nonlinear observer design with applications to Autonomous Underwater Vehicles. He is particularly interested in the design, fabrication and integration of small AUV systems. 


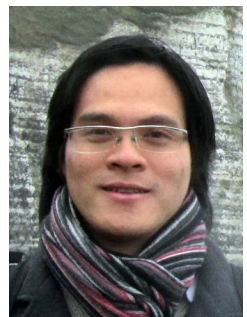

Minh-Duc Hua received the engineer's degree from Ecole Polytechnique, France in 2006 and the Ph.D. degree from the University of Nice Sophia-Antipolis, France in 2009. After two postdoctoral years with I3S laboratory in France and a short research stay with Australian National University, he joined the French National Center for Scientific Research (CNRS) in 2011, where he currently holds a tenured research position. He was assigned to the Institute for Intelligent Systems and Robotics (ISIR UPMCCNRS) in Paris from 2011 to 2016. Currently, he is with I3S UCA-CNRS Laboratory. His research interests include nonlinear control, sensor-based control, nonlinear observer, and computer vision, with applications to Unmanned Aerial Vehicles and Autonomous Underwater Vehicles. He was awarded "Outstanding Reviewer for 2017" and "Editor's Choice Paper for May 2020" of the Automatica journal. He is recipient of the "3rd Kimura Best Paper Award" of the Asian Journal of Control.

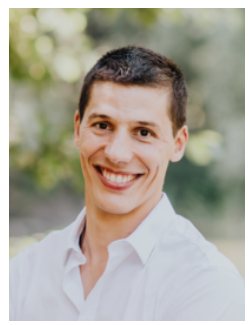

Guillaume Allibert received the M.Sc. degree from the University of Picardie Jules Verne, Amiens, France, in 2005, and the Ph.D. degree in automatic control from the University of Orleans, Orléans, France, in 2008. He is currently Associate Professor since 2009 with the Université Côte d'Azur, CNRS, I3S, France. His research interests include computer vision, nonlinear control and nonlinear observer with applications to unmanned aerial vehicles and autonomous underwater vehicles.

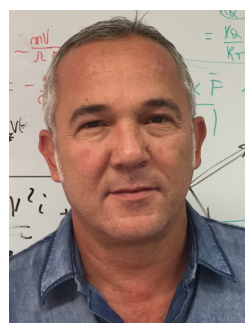

Tarek Hamel is Professor at the University of Nice Sophia Antipolis since 2003. He received his $\mathrm{Ph} . \mathrm{D}$. degree in Robotics from the University of Technology of Compiègne (UTC), France, in 1996. After two years as a research assistant at the UTC, he joined the Centre d'Etudes de Mécanique d'Ile de France in 1997 as Associate Professor. His research interests include nonlinear control theory, estimation and vision-based control with applications to Unmanned Robotic Systems. He is IEEE Fellow and senior member of the Institut Universitaire de France.

He served as Associate Editor for IEEE Transactions on Robotics, IEEE Transactions on Control System Technology and for Control Engineering Practice. 